\title{
Soft anomalous dimension matrices in heavy quark-antiquark hadroproduction in association with a gluon jet
}

\author{
E. Szarek 1 \\ Institute of Physics, Jagiellonian University \\ Eojasiewicza 11, 30-348 Kraków, Poland
}

\begin{abstract}
We compute the soft anomalous dimension (SAD) matrices for production of massive quarks $Q$ and $\bar{Q}$ in association with a gluon jet, from massless quarks $q$ and antiquarks $\bar{q}: q \bar{q} \rightarrow Q \bar{Q} g$, and in the gluon scattering $g g \rightarrow Q \bar{Q} g$. To analyze the behaviour of the eigenvalues of SAD matrices we perform numerical studies of their eigensystems at two special kinematical configurations.
\end{abstract}

${ }^{1}$ e.szarek@th.if.uj.edu.pl 


\section{Introduction}

In QCD one finds infrared divergences in perturbative corrections: soft collinear, collinear and soft non-collinear. Soft divergences appear for the energy of a gluon $E \rightarrow 0$, and the collinear ones when the angle $\theta \rightarrow 0$ between a massless parton and a gluon. After the procedure of regularization the IR divergences cancel out in observables (like cross sections), but they leave logarithmic terms depending on scales characterizing virtual and real corrections. The logarithmic remnants become very large near the absolute threshold, so they are important in processes of heavy particles production. In the absolute threshold limit the characteristic velocity of the outgoing partons $\beta$ is very small, which means that total energy $\sqrt{\hat{s}}$ of partons in center of mass system is very close to $m_{\mathrm{th}}$ where $m_{\mathrm{th}}$ is the sum of masses of products in the process. The characteristic scale of the real corrections, that come from the collinear gluon radiation is $m_{\mathrm{th}} \beta^{2}$, and the characteristic scale of the virtual corrections is proportional to $m_{\mathrm{th}}$. The real and virtual corrections combined give a leading contribution to cross section proportional to $\alpha_{s} \log ^{2} \beta^{2}$. Such logarithms appear in every order of perturbative expansion contributing with $\left(\alpha_{s} \log ^{2} \beta^{2}\right)^{n}$ in the leading logarithmic (LL) approximation, $\alpha_{s}^{n} \log ^{2 n-1} \beta^{2}$ in the next-to-leading logarithmic (NLL) approximation, and so on. When $\beta \ll 1$, $\alpha_{s} \log ^{2} \beta^{2}$ may be close or grater than one, and one needs to resum those corrections to all orders. The remnant logarithms are reordered in a new perturbative 
expansion due to the resummation procedure. The resummation formalism is described in [1, 2]. The fundamental object used in the resummation procedure is the soft anomalous dimension (SAD) matrix.

The soft gluon resummation technique that employs the SAD matrices have numerous applications in modern particle physics, in particular in estimates of superparticles hadroproduction. The SAD matrices carry information about colour flow between particles in the studied processes. The soft gluon resummation effects become very important in cross sections near the threshold for heavy particles production.

The SAD matrices were calculated for various types of processes. Firstly, calculations were performed for the Drell - Yan processes $2 \rightarrow 1$ with two incoming coloured particles and one colour-neutral [3, 4]. Then, there were considered processes $2 \rightarrow 2$, like $q \bar{q} \rightarrow q \bar{q}$ and $g g \rightarrow q \bar{q}$ for massless and massive products in final state, in one-loop approximation [2]. This approach was extended to all reactions containing light quarks and gluons [5]. It allowed to obtain predictions for cross sections for production of heavy quarks (especially for the top quark) [6, 7, 8, 9, 10, 11, 12, 13], and compare with experimental data. The SAD matrices also play an important role in predictions for squarks and gluinos hadroproduction cross sections. Soft anomalous dimension matrices were calculated at one loop [14, 15, 16] and two loops [17, 18, 19] for such processes. Recently a lot of effort 
has been made to obtain accurate predictions for reactions involving the Higgs boson. Firstly, there was obtained the hadroproduction cross section improved by the soft gluon resummation at the NNLL approximation [20] and then at the $\mathrm{N}^{3} \mathrm{LL}$ level [21] for the $2 \rightarrow 1$ process: $g g \rightarrow H^{0}$. For the supersymmetric charged Higgs boson hadroproduction the soft gluon resummation was performed at two loops for the process $b g \rightarrow t H^{-}[22$. Next the soft gluon resummation was extended to a new class of processes: $2 \rightarrow 3$ containing 4 coloured and 1 colour neutral particles, which gives more accurate predictions for the Higgs boson hadroproduction cross section in association with the top and antitop quarks [23, 24, 25, 26].

In this paper the $\mathrm{SAD}$ matrices are derived for $2 \rightarrow 3$ processes with 5 coloured particles at one loop in the perturbation theory, $q \bar{q} \rightarrow Q \bar{Q} g$ and $g g \rightarrow Q \bar{Q} g$. The quark and antiquark in the final state are both massive. Earlier calculations of the SAD matrices in similar reactions have been performed by M. Sjödahl [27, 28], however only for massless final state protons.

\section{General Formalism}

In this paper we consider the following scattering processes:

$$
q^{\alpha}\left(p_{1}\right) \bar{q}^{\beta}\left(p_{2}\right) \rightarrow Q^{\gamma}\left(p_{3}\right) \bar{Q}^{\delta}\left(p_{4}\right) g^{a}\left(p_{5}\right)
$$


and

$$
g^{a}\left(p_{1}\right) g^{b}\left(p_{2}\right) \rightarrow Q^{\alpha}\left(p_{3}\right) \bar{Q}^{\beta}\left(p_{4}\right) g^{c}\left(p_{5}\right)
$$

where $\alpha, \beta, \gamma, \delta, a, b$ and $c$ stand for colour indices (Greek letters are used for description of a fundamental representation of $\mathrm{SU}\left(N_{\mathrm{c}}\right)$ and Roman letters for an adjoint representation) and $p_{i}, i=1, \ldots, 5$, denote the momenta of particles.

Due to hard factorization theorems in QCD one can distingiush soft and hard part of sufficiently inclusive processes. The soft function $S_{I J}$ fulfills the renormalization group equation:

$$
\left(\mu \frac{\partial}{\partial \mu}+\beta(g) \frac{\partial}{\partial g}\right) S_{I J}=-\left(\Gamma_{S}^{\dagger}\right)_{I B} S_{B J}-S_{I A}\left(\Gamma_{S}\right)_{A J}
$$

where $S_{I J}$ is a matrix in colour space and carries information about soft wide angle gluon emissions, indices $I, J$ corresponds to colour tensors constructed from $\mathrm{SU}\left(N_{\mathrm{c}}\right)$ representations. They depend on a studied proccess: the colour charges of participating particles and the exchange channel. For example if we consider a quark-antiquark annihilitation, $I$ and $J$ tensors correspond to a flow of a colour singlet or octet in the s-channel. At one loop, the soft anomalous dimension matrix are defined as follows [2, 5]: 


$$
\Gamma_{S}^{\prime}(g)=-\frac{g}{2} \frac{\partial}{\partial g} \operatorname{Res}_{\epsilon \rightarrow 0} Z_{S}(g, \epsilon)
$$

where $g$ is the coupling constant for QCD, $Z_{S}(g, \epsilon)$ - a renormalization matrix of the soft matrix $S_{I J} \cdot Z_{S}$ receives contributions from the soft gluons. The general for of the SAD matrix can be derived from the paper presented in [29, 30, 31]. However, in our explicit calculations we apply the method elaborated in [2, 5].

To get $Z_{S}$ one needs to sum over the contributions $Z_{S}^{(D)}$ of relevant Feynman diagrams. Each contribution $Z_{S}^{(D)}$ to $Z_{S}$ coming from a single Feynman diagram $D$, can be factorized into a colour factor and a kinematic factor:

$$
Z_{S}^{(D)} \propto \text { colour factor } \times \text { kinematic factor }
$$

The colour part of every diagram is represented by $\mathrm{SU}\left(N_{\mathrm{c}}\right)$ tensors decomposed in an orthogonal and normalized basis. The vectors from colour basis are connected with a soft gluon line which is represented by colour tensor $i f_{a b c}$. The form of $Z_{S}$ depends on wheather the partons between which there is an exchange of the soft gluon, are massive or massless (see figure 1). For massive particles $i$ and $j$ [2, 5]:

$$
Z_{S}^{(D)}(g, \epsilon)=c^{i j} s_{i j} \frac{\alpha}{\pi} \frac{1}{\epsilon}\left(L_{\beta}^{(i j)}+L_{i}+L_{j}-1\right) .
$$

For a massive particle $i$ and a massless particle $j$ : 


$$
Z_{S}^{(D)}(g, \epsilon)=-c^{i j} s_{i j} \frac{\alpha}{2 \pi} \frac{1}{\epsilon}\left(\ln \left[\frac{v_{i j}^{2} s}{2 m_{i}^{2}}\right]-L_{i}-\ln \nu_{j}+1\right)
$$

For massless particles $i$ and $j$ :

$$
Z_{S}^{(D)}(g, \epsilon)=-c^{i j} s_{i j} \frac{\alpha}{\pi} \frac{1}{\epsilon}\left(\ln \left[\frac{\delta_{i} \delta_{j} v_{i} \cdot v_{j}}{2}\right]-\frac{1}{2} \ln \left(\nu_{i} \nu_{j}\right)+1\right) .
$$

In the above equations factors $c^{i j}$ stand for colour factors, the number $s_{i j}$ is related to the type of particles and the direction of the momentum flow in a diagram. Namely:

$$
s_{i j}=\Delta_{i} \Delta_{j} \delta_{i} \delta_{j}
$$

The factors $\Delta_{i}$ depend on the type of particles between which the exchange of the gluon occurs, they have values: $+1(-1)$ for a quark (antiquark). The factors $\delta_{i}=+1(-1)$, for the same (opposite) direction of momentum flow between a parton and the soft gluon. Vectors $v_{i}$ are rescaled momenta of the particles $v_{i}^{\mu}=\frac{p_{i}^{\mu}}{Q}$, where $Q=\sqrt{\frac{\hat{s}}{2}}$, and $v_{i j}=v_{i} \cdot v_{j}$. The factors $\nu_{i}=\frac{\left(v_{i} \cdot n\right)^{2}}{|n|^{2}}$ depend on a choice of the reference vector $n^{\mu}$ of the axial gauge. In the axial gauge $A^{0}=0$ in the center of mass system of the colliding partons one has $\nu_{i}=\frac{1}{2}$. The function $L_{\beta}^{(i j)}$ depends on the relative velocity $\beta_{i j}$ of the outgoing partons: 

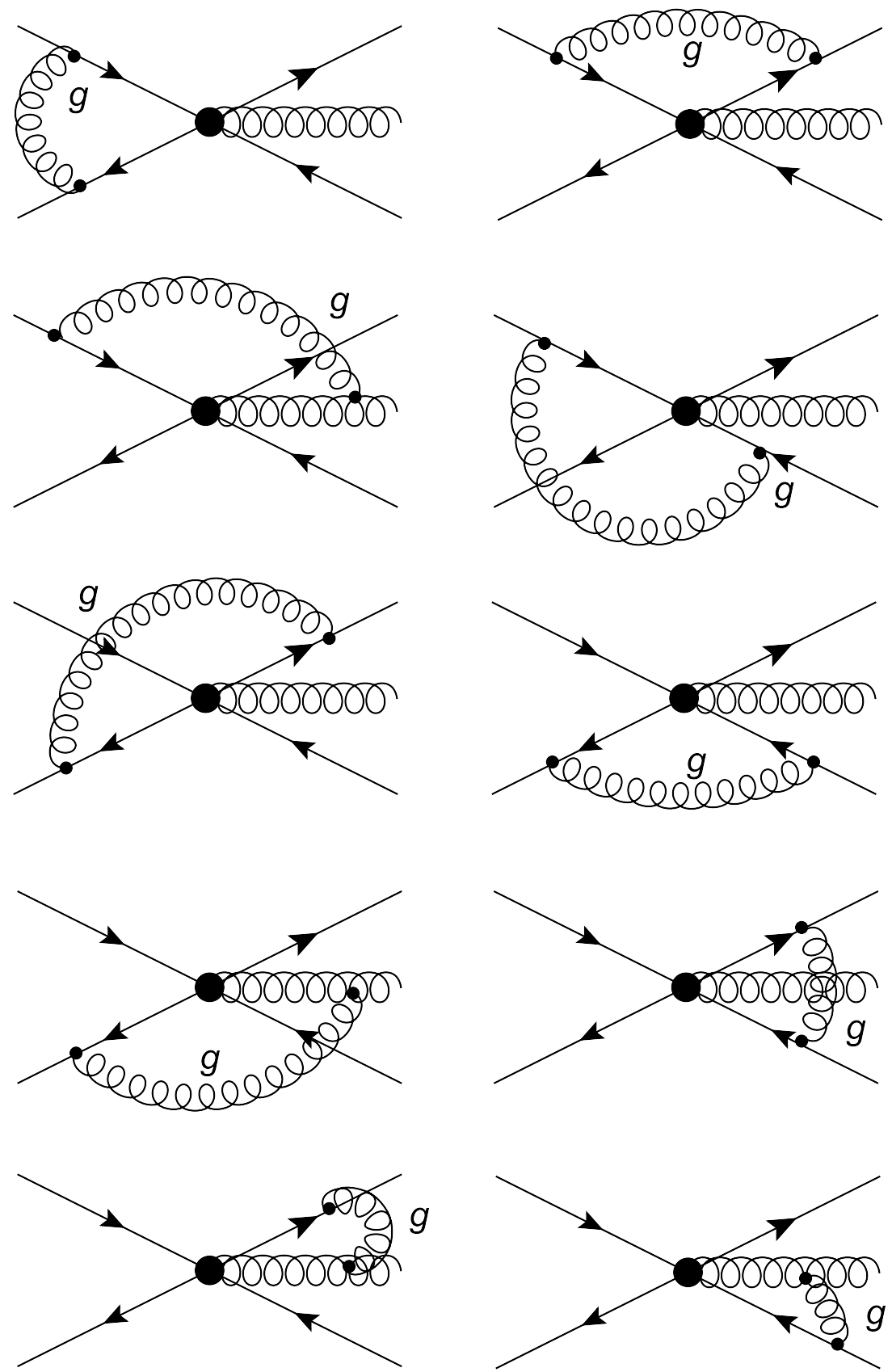

Figure 1. Feynman diagrams contributing to $Z_{S}$ for process $q \bar{q} \rightarrow Q \bar{Q} g$ (for $g g \rightarrow Q \bar{Q} g$ the topologies are analogous). The soft gluon is indicated by $g$ in the diagrams. 


$$
L_{\beta}^{(i j)}=\frac{1-2 m^{2} / \hat{s}_{i j}}{\beta_{i j}}\left(\ln \frac{1-\beta_{i j}}{1+\beta_{i j}}+i \pi\right),
$$

where $\beta_{i j}=\sqrt{1-4 m^{2} / \hat{s}_{i j}}$ and $\hat{s}_{i j}=\left(p_{i}+p_{j}\right)^{2}$. In the processes considered the massive particles are labelled by 3 and 4 , hence in what follows $\beta_{34}$ will be used. $L_{i}$ are dependent on the choice of gauge: $L_{i}=\frac{1}{2}\left[L_{i}(+n)+L_{i}(-n)\right]$, where

$$
\begin{aligned}
L_{i}( \pm n)= & \frac{1}{2} \frac{\left|v_{i} \cdot n\right|}{\sqrt{\left(v_{i} \cdot n\right)^{2}-2 m^{2} n^{2} / s}} \\
& {\left[\ln \left(\frac{\delta( \pm n) 2 m^{2} / s-\left|v_{i} \cdot n\right|-\sqrt{\left(v_{i} \cdot n\right)^{2}-2 m^{2} n^{2} / s}}{\delta( \pm n) 2 m^{2} / s-\left|v_{i} \cdot n\right|+\sqrt{\left(v_{i} \cdot n\right)^{2}-2 m^{2} n^{2} / s}}\right)\right.} \\
& \left.+\ln \left(\frac{\delta( \pm n) n^{2}-\left|v_{i} \cdot n\right|-\sqrt{\left(v_{i} \cdot n\right)^{2}-2 m^{2} n^{2} / s}}{\delta( \pm n) n^{2}-\left|v_{i} \cdot n\right|+\sqrt{\left(v_{i} \cdot n\right)^{2}-2 m^{2} n^{2} / s}}\right)\right]
\end{aligned}
$$

Contributions $L_{i}$ also appear in the self-interaction terms for the heavy quarks (antiquarks). The contribution from the self-interaction of heavy quarks (antiquarks) is

$$
\frac{\alpha_{s}}{\pi} T_{R} \frac{N_{c}^{2}-1}{N_{c}}\left(L_{i}+L_{j}-2\right) \mathbf{1}
$$

where $\mathbf{1}$ is the identity matrix in the colour space and the factor $T_{R}$ comes from the normalization of generators and it equals $\frac{1}{2}$. The contribution from the selfinteraction of heavy quarks (antiquarks) is added to the soft anomalous dimension 
matrix and the dependence of $\Gamma_{S}^{\prime}$ on $L_{i}$ is canceled out. Following refs. [2, 5] the Drell - Yan contribution is subtracted from the soft anomalous dimension matrix. At one loop the Drell - Yan SAD matrix takes the form $\frac{\alpha_{s}}{\pi} C_{F} \mathbf{1}\left(\frac{\alpha_{s}}{\pi} C_{A} \mathbf{1}\right)$ for the partons in the colour triplet (octet) state and $C_{A}=T_{R} 2 N_{c}$ and $C_{F}=T_{R} \frac{N_{c}^{2}-1}{N_{c}}$. The final form of the soft anomalous dimension matrix $\Gamma_{S}(g, \epsilon)$ is the following:

$$
\Gamma_{S}(g, \epsilon)=\Gamma_{S}^{\prime}(g, \epsilon)+\frac{\alpha_{s}}{\pi} T_{R} \frac{N_{c}^{2}-1}{N_{c}}\left(L_{3}+L_{4}-2\right) \mathbf{1}-\frac{1}{2} \sum_{i} C_{A, F}^{i} \mathbf{1},
$$

where $i$ - all massless particles in the examined process.

In this paper processes with five interacting particles are considered (see figure $2)$.

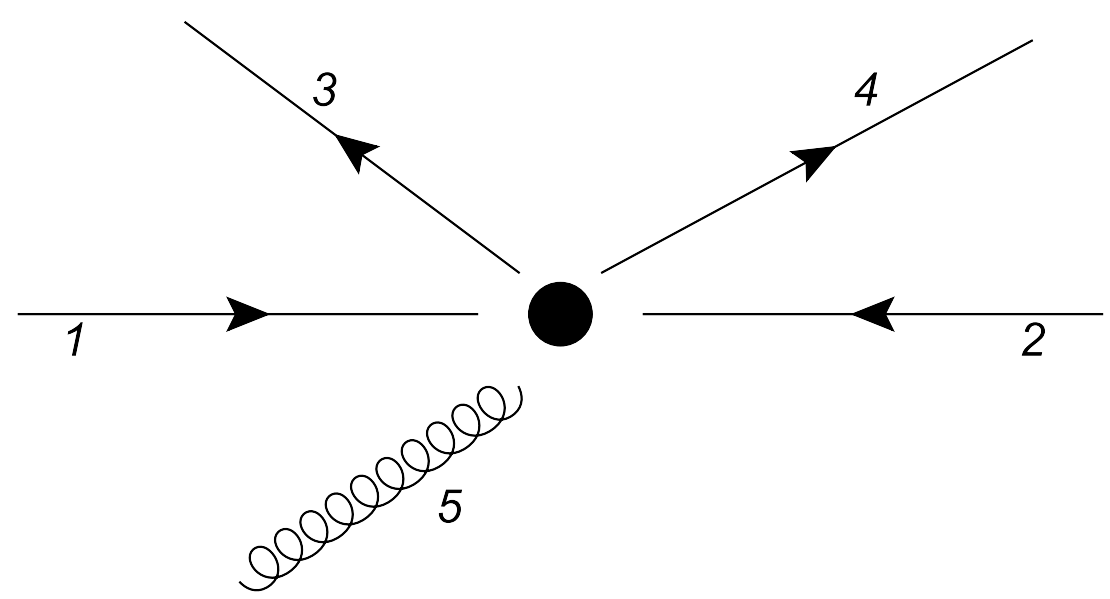

Figure 2. An example of a particle collision in a process $2 \rightarrow 3$.

To fully describe the phase space of such physical system one needs five inde- 
pendent variables: the global azimuthal angle $\phi$, which carries information about the rotation symmetry of the reaction and four Mandelstam-type variables:

$$
\begin{aligned}
& t_{1}=\left(p_{3}-p_{1}\right)^{2}, \\
& t_{2}=\left(p_{4}-p_{2}\right)^{2}, \\
& u_{1}=\left(p_{3}-p_{2}\right)^{2}, \\
& u_{2}=\left(p_{4}-p_{1}\right)^{2} .
\end{aligned}
$$

The remaining scalar products of particle momenta $p_{i} \cdot p_{5}(i=1,2,3,4)$ may be expressed in the terms of above variables:

$$
\begin{aligned}
& p_{1} \cdot p_{5}=\frac{1}{2}\left(t_{1}+u_{2}+s-m_{3}^{2}-m_{4}^{2}\right), \\
& p_{2} \cdot p_{5}=\frac{1}{2}\left(t_{2}+u_{1}+s-m_{3}^{2}-m_{4}^{2}\right), \\
& p_{3} \cdot p_{5}=\frac{1}{2}\left(t_{2}+u_{2}+s-m_{3}^{2}-m_{4}^{2}\right), \\
& p_{4} \cdot p_{5}=\frac{1}{2}\left(t_{1}+u_{1}+s-m_{3}^{2}-m_{4}^{2}\right),
\end{aligned}
$$

where $m_{3,4}$ is the mass of heavy quark (antiquark). 


\section{Results}

In this section we collect results for the soft anomalous dimension matrices for two processes $q \bar{q} \rightarrow Q \bar{Q} g$ and $g g \rightarrow Q \bar{Q} g$, where $q, \bar{q}-$ denote the massless quark/antiquark, and $Q, \bar{Q}$ - the massive quark/antiquark. Calculations of the colour factors were obtained in the $s$-channel basis, using the package ColorMath [28] for Mathematica. The colour factors were combined with formulas (44), (6) (8), also (13), and $\Gamma_{S}$ was obtained. It is convenient to introduce new variables $T_{1}, T_{2}, U_{1}, U_{2}$ and then $\Lambda, \Omega, \Gamma, \Sigma$, which are defined in the following way:

$$
\begin{aligned}
& T_{1}=\ln \left(\frac{2 p_{1} \cdot p_{3}}{m_{3} \sqrt{s}}\right)-\frac{1-i \pi}{2} \\
& T_{2}=\ln \left(\frac{2 p_{2} \cdot p_{4}}{m_{4} \sqrt{s}}\right)-\frac{1-i \pi}{2} \\
& U_{1}=\ln \left(\frac{2 p_{2} \cdot p_{3}}{m_{4} \sqrt{s}}\right)-\frac{1-i \pi}{2} \\
& U_{2}=\ln \left(\frac{2 p_{1} \cdot p_{4}}{m_{3} \sqrt{s}}\right)-\frac{1-i \pi}{2} .
\end{aligned}
$$

In this study the case is considered of the mass of quark and antiquark that have the same value $m=m_{3}=m_{4}$ and we introduce variables, 


$$
\begin{aligned}
\Lambda & =T_{1}+T_{2}+U_{1}+U_{2}, \\
\Omega & =T_{1}+T_{2}-U_{1}-U_{2}, \\
\Gamma & =T_{1}-T_{2}+U_{1}-U_{2}, \\
\Sigma & =T_{1}-T_{2}-U_{1}+U_{2} .
\end{aligned}
$$

In what follows, the SAD matrices will be presented in terms of the independent variables $\Lambda, \Omega, \Gamma, \Sigma$ and the variables $v_{i 5}=2 p_{i} \cdot p_{5} / s$ that can be expressed by $\Lambda$, $\Omega, \Gamma, \Sigma$ with equalities (11), (12) and (13). The variables $v_{i 5}$ are kept in order to simplify the form of the matrices.

\section{$3.1 \quad q \bar{q} \rightarrow Q \bar{Q} g$}

The following orthogonal and normalized colour basis was used in the calculations [27]:

$$
\begin{aligned}
T_{\alpha \beta \gamma \zeta a}^{1} & =\frac{1}{\sqrt{N_{c}\left(N_{c}^{2}-1\right) T_{R}}} \delta_{\alpha \beta} t_{\gamma \zeta}^{a}, \\
T_{\alpha \beta \gamma \zeta a}^{2} & =\frac{1}{\sqrt{N_{c}\left(N_{c}^{2}-1\right) T_{R}}} \delta_{\gamma \zeta} t_{\beta \alpha}^{a}, \\
T_{\alpha \beta \gamma \zeta a}^{3} & =\frac{1}{\sqrt{2 N_{c}\left(N_{c}^{2}-1\right)\left(T_{R}\right)^{3}}} t_{\beta \alpha}^{b} t_{\gamma \zeta}^{c} i f_{b c a},
\end{aligned}
$$




$$
T_{\alpha \beta \gamma \zeta a}^{4}=\frac{\sqrt{N_{c}}}{\sqrt{2\left(N_{c}^{2}-4\right)\left(N_{c}^{2}-1\right)\left(T_{R}\right)^{3}}} t_{\beta \alpha}^{b} t_{\gamma \zeta}^{c} d_{b c a} .
$$

Notice that indices of the adjoint representation are $a, b, c$ and indices of the fundamental representation are $\alpha, \beta, \gamma$ and $\zeta$. The following results are valid for $N_{c} \geq 3[27$.

The soft anomalous dimension matrix $\Gamma_{q \bar{q} \rightarrow Q \bar{Q} g}$ can be split into two parts:

$$
\Gamma_{q \bar{q} \rightarrow Q \bar{Q} g}=\Gamma_{q \bar{q} \rightarrow Q \bar{Q} g}^{(1)}(\Lambda, \Omega, \Gamma, \Sigma)+\Gamma_{q \bar{q} \rightarrow Q \bar{Q} g}^{(2)}\left(v_{i 5}\right),
$$

where $\Gamma^{(1)}$ receives contributions from the soft gluon exchanges between particles $1,2,3,4$, and $\Gamma^{(2)}$ from exchanges between particles $i$ and 5 , with $i=1,2,3,4$. Hence:

$$
\begin{aligned}
& \Gamma_{q \bar{q} \rightarrow Q \bar{Q} g}^{(1)}=\frac{\alpha_{s}}{\pi} T_{R} \times \\
& \left(\begin{array}{cccc}
\frac{1+\left(1-N_{c}^{2}\right) L_{\beta}}{N_{c}} & \frac{\Omega}{N_{c}} & \frac{\sqrt{N_{c}^{2}-4}}{\sqrt{2} N_{c}} \Omega & \frac{\Gamma}{\sqrt{2}} \\
\frac{\Omega}{N_{c}} & \frac{2+2 L_{\beta}+N_{c}^{2}}{2 N_{c}} & \frac{\sqrt{N_{c}^{2}-4}}{\sqrt{2} N_{c}} \Omega & \frac{\Sigma}{\sqrt{2}} \\
\frac{\sqrt{N_{c}^{2}-4}}{\sqrt{2} N_{c}} \Omega & \frac{\sqrt{N_{c}^{2}-4}}{\sqrt{2} N_{c}} \Omega & \frac{4+4 L_{\beta}+\left(N_{c}^{2}-12\right) \Omega+N_{c}^{2}(2+\Lambda)}{4 N_{c}} & \frac{1}{4} \sqrt{N_{c}^{2}-4}(\Gamma+\Sigma) \\
\frac{\Gamma}{\sqrt{2}} & \frac{\Sigma}{\sqrt{2}} & \frac{1}{4} \sqrt{N_{c}^{2}-4}(\Gamma+\Sigma) & \frac{4+4 L_{\beta}+\left(N_{c}^{2}-4\right) \Omega+N_{c}^{2}(2+\Lambda)}{4 N_{c}}
\end{array}\right),
\end{aligned}
$$

and 


$$
\begin{aligned}
& \Gamma_{q \bar{q} \rightarrow Q \bar{Q} g}^{(2)}=\frac{\alpha_{s}}{\pi} T_{R} \times \\
& \left(\begin{array}{cccc}
N_{c} \ln \left(v_{15} v_{25}\right) & 0 & 0 & \frac{1}{\sqrt{2}} \ln \left(\frac{v_{45}}{v_{35}}\right) \\
0 & \frac{1}{2} N_{c} \ln \left(v_{35} v_{45}\right) & 0 & \ln \left(\frac{v_{25}}{v_{15}}\right) \sqrt{2} \\
0 & 0 & \frac{1}{4} N_{c} \ln \left(v_{15}^{2} v_{25}^{2} v_{35} v_{45}\right) & \frac{1}{4} \ln \left(\frac{v_{25}^{2} v_{45}}{v_{15}^{2} v_{35}} \sqrt{N_{c}^{2}-4}\right. \\
\frac{1}{\sqrt{2}} \ln \left(\frac{v_{45}}{v_{35}}\right) & \ln \left(\frac{v_{25}}{v_{15}}\right) \sqrt{2} & \frac{1}{4} \ln \left(\frac{v_{25}^{2} v_{45}}{v_{15}^{2} v_{35}}\right) \sqrt{N_{c}^{2}-4} & \frac{1}{4} N_{c} \ln \left(v_{15}^{2} v_{25}^{2} v_{35} v_{45}\right)
\end{array}\right) \\
& +\quad \frac{\alpha_{s}}{\pi} T_{R} \times \operatorname{diag}\left(\frac{i \pi}{N_{c}}, i \pi \frac{1-N_{c}^{2}}{N_{c}}, i \pi \frac{2-N_{c}^{2}}{2 N_{c}}, i \pi \frac{2-N_{c}^{2}}{2 N_{c}}\right) .
\end{aligned}
$$

The SAD matrices for $q \bar{q} \rightarrow Q \bar{Q} g$ were calculated in parallel in $[33]^{2}$. Note that, the obtained SAD matrix is complex symmetric. This property has been proved to hold in general in an orthonormal basis [32]. The same feature will be found also for the $g g$-channel. For clarity we denoted $L_{\beta}^{(34)}$ as $L_{\beta}$ in all the matrices. The general form of the soft anomalous dimension matrix is rather complicated, hence in order to provide more insight into its properties we consider special kinematical configurations for which the matrix simplifies. First, we consider the case when the momenta of the outgoing quark and antiquark are equal, $p_{3}^{\mu}=p_{4}^{\mu}$. Then variables $\Lambda, \Omega, \Gamma, \Sigma$ reduce to $\Lambda \rightarrow \Lambda^{\prime}=2 T_{1}+2 U_{1}, \Sigma \rightarrow \Sigma^{\prime}=2 T_{1}-2 U_{1}, \Gamma \rightarrow 0, \Omega \rightarrow 0$. In this special case it is convenient to introduce a variable $\beta=\sqrt{1-\frac{4 m^{2}}{\hat{s}}}$. In this limit the form of the soft anomalous matrix becomes significantly simpler:

\footnotetext{
${ }^{2}$ Several differences were found between results of 33 and the results of this paper and their origin was clarified in correspondence with R. Schäfer.
} 


$$
\begin{aligned}
& \Gamma_{q \bar{q} \rightarrow Q \bar{Q} g}^{(1)}\left(p_{3}^{\mu}=p_{4}^{\mu}\right)=\frac{\alpha_{s}}{\pi} T_{R} \times \\
& \left(\begin{array}{cccc}
\frac{1+\left(1-N_{c}^{2}\right) L_{\beta}}{N_{c}} & 0 & 0 & 0 \\
0 & \frac{2+2 L_{\beta}+N_{c}^{2}}{2 N_{c}} & 0 & \frac{\Sigma^{\prime}}{\sqrt{2}} \\
0 & 0 & \frac{4+4 L_{\beta}+N_{c}^{2}\left(2+\Lambda^{\prime}\right)}{4 N_{c}} & \frac{\Sigma^{\prime}}{4} \sqrt{N_{c}^{2}-4} \\
0 & \frac{\Sigma^{\prime}}{\sqrt{2}} & \frac{\Sigma^{\prime}}{4} \sqrt{N_{c}^{2}-4} & \frac{4+4 L_{\beta}+N_{c}^{2}\left(2+\Lambda^{\prime}\right)}{4 N_{c}}
\end{array}\right) \\
& \Gamma_{q \bar{q} \rightarrow Q \bar{Q} g}^{(2)}\left(p_{3}^{\mu}=p_{4}^{\mu}\right)=\frac{\alpha_{s}}{\pi} T_{R} \times \\
& \left(\begin{array}{cccc}
N_{c} \ln \left(v_{15} v_{25}\right) & 0 & 0 & 0 \\
0 & N_{c} \ln v_{35} & 0 & \ln \left(\frac{v_{25}}{v_{15}}\right) \sqrt{2} \\
0 & 0 & \frac{1}{2} N_{c} \ln \left(v_{15} v_{25} v_{35}\right) & \frac{1}{2} \ln \left(\frac{v_{25}}{v_{15}}\right) \sqrt{N_{c}^{2}-4} \\
0 & \ln \left(\frac{v_{25}}{v_{15}}\right) \sqrt{2} & \frac{1}{2} \ln \left(\frac{v_{25}}{v_{15}}\right) \sqrt{N_{c}^{2}-4} & \frac{1}{2} N_{c} \ln \left(v_{15} v_{25} v_{35}\right)
\end{array}\right) \\
& +\frac{\alpha_{s}}{\pi} T_{R} \times \operatorname{diag}\left(\frac{i \pi}{N_{c}}, i \pi \frac{1-N_{c}^{2}}{N_{c}}, i \pi \frac{2-N_{c}^{2}}{2 N_{c}}, i \pi \frac{2-N_{c}^{2}}{2 N_{c}}\right) .
\end{aligned}
$$

It can be seen that the soft anomalous dimension matrix can be divided in two blocks: $1 \times 1$ and $3 \times 3$.

Next the limit $\Sigma^{\prime}=0$ is performed that corresponds to $p_{1} \cdot p_{3}=p_{2} \cdot p_{3}$. The obtained matrix has a diagonal form: 


$$
\begin{aligned}
& \Gamma_{q \bar{q} \rightarrow Q \bar{Q} g}\left(\Sigma^{\prime}=0\right)=\frac{\alpha_{s}}{\pi} T_{R} \times \\
& \left\{\frac{1}{N_{c}} \times \operatorname{diag}\left(1+\left(1-N_{c}^{2}\right) L_{\beta}, 1+L_{\beta}+\frac{N_{c}^{2}}{2}, 1+L_{\beta}+\frac{N_{c}^{2}}{4}\left(2+\Lambda^{\prime}\right), 1+L_{\beta}+\frac{N_{c}^{2}}{4}\left(2+\Lambda^{\prime}\right)\right)\right. \\
& +\operatorname{diag}\left(2 N_{c} \ln v_{15}, N_{c} \ln v_{35}, \frac{1}{2} N_{c} \ln \left(v_{15}^{2} v_{35}\right), \frac{1}{2} N_{c} \ln \left(v_{15}^{2} v_{35}\right)\right) \\
& \left.+\operatorname{diag}\left(\frac{i \pi}{N_{c}}, i \pi \frac{1-N_{c}^{2}}{N_{c}}, i \pi \frac{2-N_{c}^{2}}{2 N_{c}}, i \pi \frac{2-N_{c}^{2}}{2 N_{c}}\right)\right\}
\end{aligned}
$$

\subsubsection{Analysis of the eigenvalues for $q \bar{q} \rightarrow Q \bar{Q} g$}

In this section we consider the behaviour of the SAD eigenvalues for $p_{3}=p_{4}$ and two different scattering angles $\theta\left(90^{\circ}\right.$ and $\left.30^{\circ}\right)$ where $\theta$ is an angle between the incoming and outgoing partons in the CMS frame. $\theta=90^{\circ}$ represents the most symmetrical case, and the choice of $\theta=30^{\circ}$ represents a less symmetrical configuration. The limit $\Sigma^{\prime} \rightarrow 0$ corresponds to the case of $\theta=90^{\circ}$. This analysis must be done carrefully because $L_{\beta}^{(34)}$ in the limit $\beta_{34} \rightarrow 0$ gives singular terms $\propto \frac{i}{\beta_{34}}$. We need to execute three steps. While performing the limit $\beta_{34} \rightarrow 0$ one subtracts the singular terms from the $\mathrm{SAD}$ matrix and then the limit $\beta \rightarrow 0$ may be studied. Finally we present results after a subtraction of the asymptotic small $\beta$ behaviour that is treated analytically. Numerical calculations were performed

for the $N_{c}=3$ case. The eigenvalues of $\tilde{\Gamma}_{S}$ does not contain the prefactor $\frac{\alpha_{s}}{\pi}$. 
The relation between the full SAD matrix $\Gamma_{S}$ and $\tilde{\Gamma}_{S}$ is $\Gamma_{S}=\frac{\alpha_{s}}{\pi} \tilde{\Gamma}_{S}$. The singular matrix subtracted from $\tilde{\Gamma}_{S}$ has a form $\frac{i \pi}{\beta_{34}} \times \operatorname{diag}\left(-\frac{2}{3}, \frac{1}{12}, \frac{1}{12}, \frac{1}{12}\right)$.

For a general $\beta$ there is a degeneracy of eigenvalues for $\theta=90^{\circ}$, there are three different eigenvalues. For $\theta=30^{\circ}$ there is no degeneracy. All eigenvalues are complex with non-trival real and imaginary part. For $\theta=90^{\circ}$ there are two different values of the imaginary part of eigenvalues instead of four, which is the case of $30^{\circ}$.

For $\beta \rightarrow 0$ one finds a singular term proportional to $\log \beta$ which gives a contribution to the real part of the eigenvalues. Each eigenvalue of $\Gamma_{S}$ has the same leading behaviour in $\beta \rightarrow 0$ for both scattering angles. One finds one asymptotic form of the eigenvalues of small $\beta$ :

$$
\lambda^{\operatorname{sing}}=6 \log \beta
$$

In Figures 3 and 4 we show regularized eigenvalues. They are defined as $\lambda_{i}^{\text {reg }}=$ $\lambda_{i}-\lambda^{\text {sing }}$. One observes a quite similar behaviour of the regularized eigensystem for $\theta=90^{\circ}$ and $\theta=30^{\circ}$. In Figure 3 one can see that all $\operatorname{Re}\left(\lambda^{\mathrm{reg}, 90^{\circ}}\right)$ are either constant $\left(\lambda_{1}\right)$ or slightly increasing $\left(\lambda_{2}, \lambda_{34}\right)$ up to $\beta \approx 0.4 . \operatorname{Im}\left(\lambda^{90^{\circ}}\right)$ are constant in $\beta$. In the case of $\theta=30^{\circ}$, the real and imaginary part of $\lambda_{1}$ shows a constant behaviour. The real parts of $\lambda_{2}, \lambda_{3}$ and $\lambda_{4}$ exhibit a similar behaviour for small $\beta$ - they are slowly varying for moderate $\beta$, then for $\beta>0.6$ they are rapidly increasing. The 
imaginary parts of these remaining eigenvalues are nearly constant for $\beta<0.7$, and then start to slowly decrease $\left(\lambda_{2}\right.$ and $\left.\lambda_{3}\right)$ or slowly increase $\left(\lambda_{4}\right)$. 

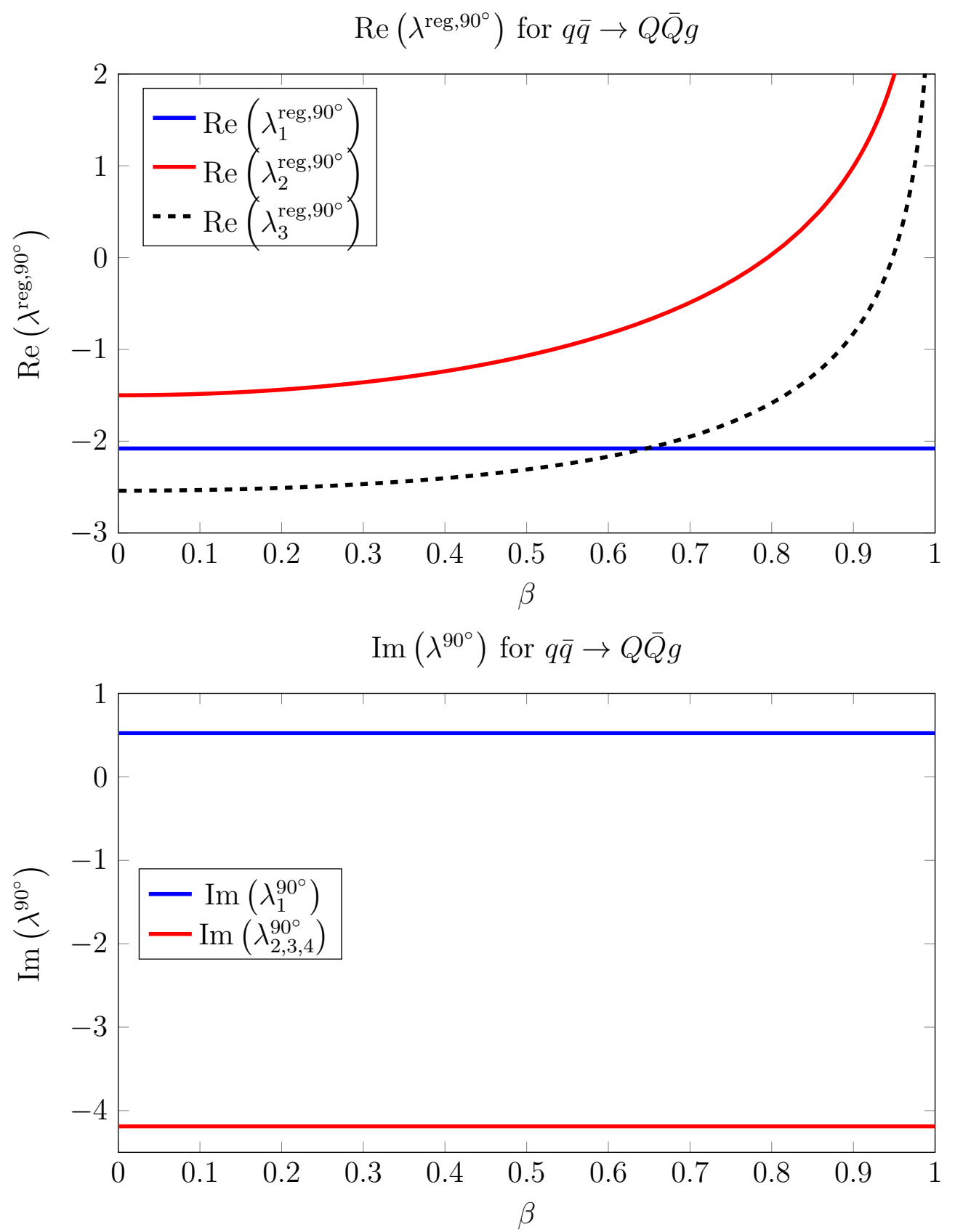

Figure 3. The real (top) and imaginary (bottom) parts of the eigenvalues of $\tilde{\Gamma}_{S}$ for $q \bar{q} \rightarrow Q \bar{Q} g$ at $\theta=90^{\circ}$. 

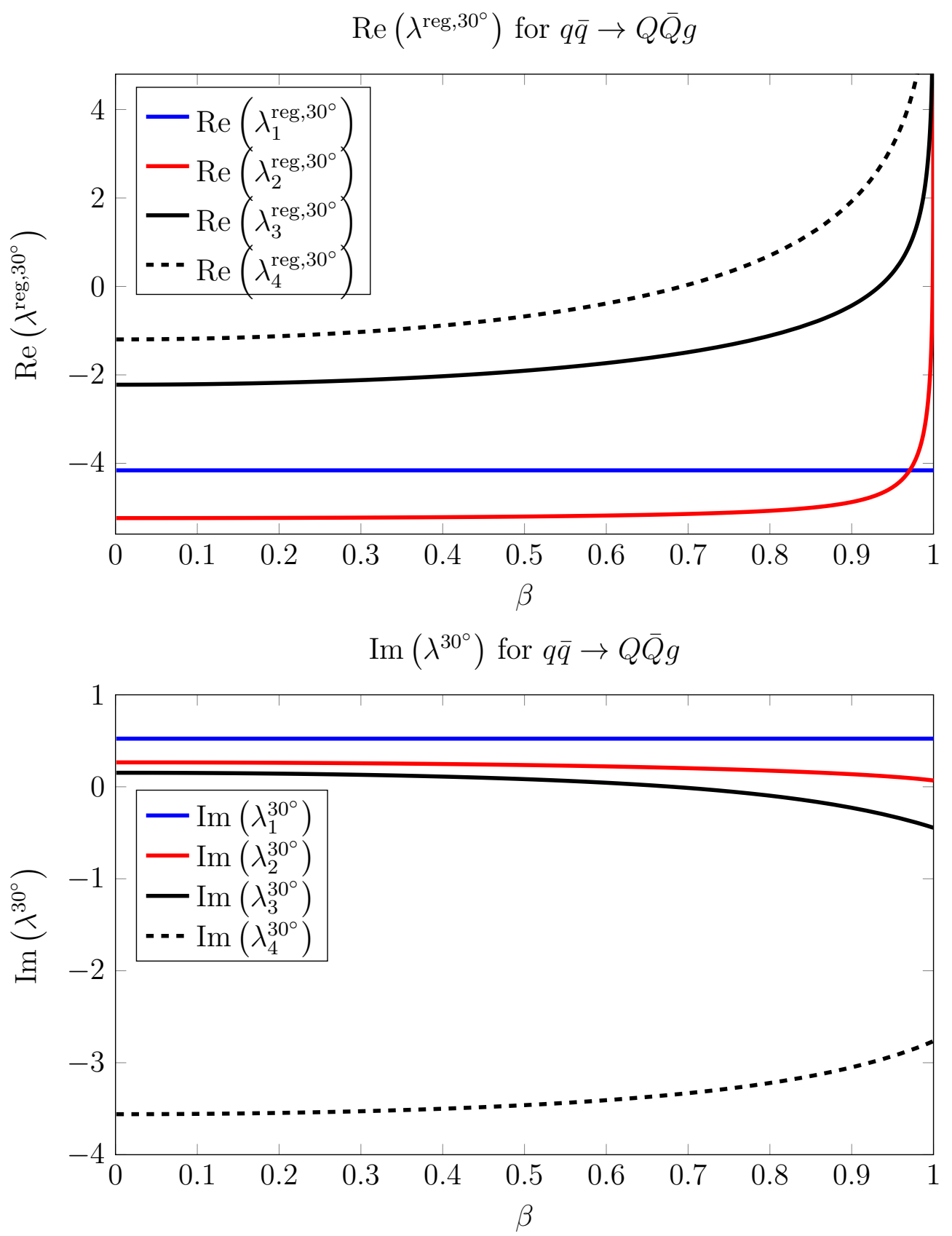

Figure 4. The real (top) and imaginary (bottom) parts of the eigenvalues of $\tilde{\Gamma}_{S}$ for $q \bar{q} \rightarrow Q \bar{Q} g$ at $\theta=30^{\circ}$. 


\section{$3.2 \quad g g \rightarrow Q \bar{Q} g$}

The following orthogonal and normalized colour basis was used in the calculations

[27]:

$$
\begin{aligned}
& T_{a b \alpha \beta c}^{1}=\frac{1}{\left(N_{c}^{2}-1\right) \sqrt{T_{R}}} t_{\alpha \beta}^{c} \delta_{a b}, \\
& T_{a b \alpha \beta c}^{2}=\frac{1}{N_{c} \sqrt{2\left(N_{c}^{2}-1\right) T_{R}}} i f_{a b c} \delta_{\alpha \beta}, \\
& T_{a b \alpha \beta c}^{3}=\frac{1}{\sqrt{2\left(N_{c}^{2}-4\right)\left(N_{c}^{2}-1\right) T_{R}}} d_{a b c} \delta_{\alpha \beta}, \\
& T_{a b \alpha \beta c}^{4}=\frac{1}{2 N_{c} \sqrt{\left(N_{c}^{2}-1\right)\left(T_{R}\right)^{3}}} i f_{a b n} i f_{m c n} t_{\alpha \beta}^{m}, \\
& T_{a b \alpha \beta c}^{5}=\frac{1}{\sqrt{4\left(N_{c}^{2}-4\right)\left(N_{c}^{2}-1\right)\left(T_{R}\right)^{3}}} d_{a b n} i f_{m c n} t_{\alpha \beta}^{m}, \\
& T_{a b \alpha \beta c}^{6}=\frac{1}{\sqrt{4\left(N_{c}^{2}-4\right)\left(N_{c}^{2}-1\right)\left(T_{R}\right)^{3}}} i f_{a b n} d_{m c n} t_{\alpha \beta}^{m}, \\
& T_{a b \alpha \beta c}^{7}=\frac{1}{\sqrt{4\left(N_{c}^{2}-4\right)^{2}\left(N_{c}^{2}-1\right)\left(T_{R}\right)^{3}}} d_{a b n} d_{m c n} t_{\alpha \beta}^{m}, \\
& T_{a b \alpha \beta c}^{8}=\frac{1}{\sqrt{2\left(N_{c}^{2}-4\right)\left(N_{c}^{2}-1\right)\left(T_{R}\right)^{3}}} P_{a b m c}^{10+10} t_{\alpha \beta}^{m}, \\
& T_{a b \alpha \beta c}^{9}=\frac{1}{\sqrt{2\left(N_{c}^{2}-4\right)\left(N_{c}^{2}-1\right)\left(T_{R}\right)^{3}}} P_{a b m c}^{10-10} t_{\alpha \beta}^{m}, \\
& T_{a b \alpha \beta c}^{10}=\frac{-1}{\sqrt{N_{c}^{2}\left(N_{c}+3\right)\left(N_{c}-1\right)\left(T_{R}\right)^{3}}} P_{a b m c}^{27} t_{\alpha \beta}^{m}, \\
& T_{a b \alpha \beta c}^{11}=\frac{1}{\sqrt{N_{c}^{2}\left(N_{c}-3\right)\left(N_{c}+1\right)\left(T_{R}\right)^{3}}} P_{a b m c}^{0} t_{\alpha \beta}^{m},
\end{aligned}
$$

where 


$$
\begin{aligned}
P_{a b c d}^{10+\overline{0}}= & \frac{1}{2}\left(\delta_{a c} \delta_{b d}-\delta_{a d} \delta_{c b}\right)-\frac{1}{N_{c}} f_{a b g} f_{c d g} \\
P_{a b c d}^{10-\overline{10}}= & \frac{1}{2} d_{a c g} i f_{b g d}-\frac{1}{2} d_{b g d} i f_{a c g} \\
P_{a b c d}^{27}= & \frac{N_{c}}{4\left(N_{c}+2\right)} d_{a b g} d_{c d g}+\frac{1}{2} f_{a d g} f_{c b g}-\frac{1}{4} f_{a b g} f_{c d g}+\frac{1}{4} \delta_{a d} \delta_{b c}+\frac{1}{4} \delta_{a c} \delta_{b d} \\
& +\frac{1}{2\left(N_{c}+1\right)} \delta_{a b} \delta_{c d}, \\
P_{a b c d}^{0}= & -\frac{N_{c}}{4\left(N_{c}+2\right)} d_{a b g} d_{c d g}-\frac{1}{2} f_{a d g} f_{c b g}+\frac{1}{4} f_{a b g} f_{c d g}+\frac{1}{4} \delta_{a d} \delta_{b c}+\frac{1}{4} \delta_{a c} \delta_{b d} \\
& -\frac{1}{2\left(N_{c}+1\right)} \delta_{a b} \delta_{c d} .
\end{aligned}
$$

As for the $q \bar{q} \rightarrow Q \bar{Q} g$ case the soft anomalous dimension matrix $\Gamma_{g g \rightarrow Q \bar{Q} g}$ is split in two parts:

$$
\Gamma_{g g \rightarrow Q \bar{Q} g}=\Gamma_{g g \rightarrow Q \bar{Q} g}^{(1)}(\Lambda, \Omega, \Gamma, \Sigma)+\Gamma_{g g \rightarrow Q \bar{Q} g}^{(2)}\left(v_{i 5}\right),
$$

where 
$\Gamma_{g g \rightarrow Q \bar{Q} g}^{(1)}=\frac{\alpha_{s}}{\pi} T_{R} \times$

\begin{tabular}{|c|c|c|c|c|c|}
\hline$\frac{1+\left(1-N_{c}^{2}\right) L_{\beta}}{N_{c}}$ & $-\Omega$ & 0 & $-\frac{\Omega}{\sqrt{2}}$ & $\frac{\Gamma}{\sqrt{2}}$ & 0 \\
\hline$-\Omega$ & $\frac{2+2 L_{\beta}+N_{c}^{2}}{N_{c}}$ & 0 & 0 & 0 & $\frac{\Gamma}{2}$ \\
\hline 0 & 0 & $\frac{1+\left(1-N_{c}^{2}\right) L_{\beta}}{N_{c}}$ & 0 & 0 & 0 \\
\hline$-\frac{\Omega}{\sqrt{2}}$ & 0 & 0 & $\frac{4+4 L_{\beta}+N_{c}^{2}(2+\Lambda)}{4 N_{c}}$ & $-\frac{\Sigma N_{c}}{4}$ & 0 \\
\hline$\frac{\Gamma}{\sqrt{2}}$ & 0 & 0 & $-\frac{\Sigma N_{c}}{4}$ & $\frac{4+4 L_{\beta}+N_{c}^{2}(2+\Lambda)}{4 N_{c}}$ & $-\frac{\Omega}{\sqrt{2}}$ \\
\hline 0 & $\frac{\Gamma}{2}$ & 0 & 0 & $\frac{-\Omega}{\sqrt{2}}$ & $\frac{2+2 L_{\beta}+N_{c}^{2}(2+\Lambda)}{2 N_{c}}$ \\
\hline 0 & $\frac{\Omega}{2 \sqrt{2}} \sqrt{\frac{\left(N_{c}-3\right)\left(N_{c}-1\right)\left(N_{c}+2\right)}{\left(N_{c}-2\right)}}$ & $-\Omega \sqrt{\frac{N_{c}+1}{2\left(N_{c}-1\right)}}$ & $\frac{\Omega}{2} \sqrt{\frac{\left(N_{c}-3\right)\left(N_{c}+2\right)}{\left(N_{c}-2\right)\left(N_{c}-1\right)}}$ & 0 & $\frac{\Sigma}{2 \sqrt{2}} \sqrt{\frac{\left(N_{c}-3\right)\left(N_{c}-1\right)\left(N_{c}+2\right)}{\left(N_{c}-2\right)}}$ \\
\hline 0 & $-\frac{\Omega \sqrt{2}}{\sqrt{N_{c}^{2}-4}}$ & $\frac{-\Omega}{\sqrt{2}}$ & $-\frac{\Omega\left(N_{c}^{2}-12\right)}{4 \sqrt{N_{c}^{2}-4}}$ & $\frac{\Gamma}{4} \sqrt{N_{c}^{2}-4}$ & $\frac{N_{c} \Sigma}{\sqrt{2} \sqrt{N_{c}^{2}-4}}$ \\
\hline 0 & $\frac{\Omega}{2 \sqrt{2}} \sqrt{\frac{\left(N_{c}+3\right)\left(N_{c}+1\right)\left(N_{c}-2\right)}{\left(N_{c}+2\right)}}$ & $-\Omega \sqrt{\frac{N_{c}+3}{2\left(N_{c}+1\right)}}$ & $\frac{-\Omega}{2} \sqrt{\frac{\left(N_{c}-2\right)\left(N_{c}+3\right)}{\left(N_{c}+1\right)\left(N_{c}+2\right)}}$ & 0 & $\frac{-\Sigma}{2 \sqrt{2}} \sqrt{\frac{\left(N_{c}-2\right)\left(N_{c}+3\right)}{\left(N_{c}+1\right)\left(N_{c}+2\right)}}$ \\
\hline 0 & 0 & $\frac{\Gamma}{\sqrt{2}}$ & $\frac{\Gamma}{4} \sqrt{N_{c}^{2}-4}$ & $\frac{-\Gamma}{4} \sqrt{N_{c}^{2}-4}$ & 0 \\
\hline 0 & 0 & $\frac{-\Omega \sqrt{2}}{\sqrt{N_{c}^{2}-1}}$ & $\Omega \sqrt{\frac{N_{c}^{2}-4}{N_{c}^{2}-1}}$ & 0 & 0 \\
\hline
\end{tabular}

(29)

$$
\begin{array}{lc}
\ldots & 0 \\
\ldots & \frac{\Omega}{2 \sqrt{2}} \sqrt{\frac{\left(N_{c}-3\right)\left(N_{c}-1\right)\left(N_{c}+2\right)}{\left(N_{c}-2\right)}} \\
\ldots & -\Omega \sqrt{\frac{N_{c}+1}{2\left(N_{c}-1\right)}} \\
\ldots & \frac{\Omega}{2} \sqrt{\frac{\left(N_{c}-3\right)\left(N_{c}+2\right)}{\left(N_{c}-2\right)\left(N_{c}-1\right)}} \\
\ldots & 0 \\
\ldots & -\frac{\Sigma N_{c}}{4} \\
\ldots & \frac{4+4 L_{\beta}+N_{c}^{2}(3+\Lambda)}{4 N_{c}} \\
\ldots & 0 \\
\ldots & 0 \\
\ldots & \frac{-\Sigma}{2} \sqrt{\frac{N_{c}-3}{N_{c}-1}} \\
\ldots & 0
\end{array}
$$$$
\begin{gathered}
0 \\
-\frac{2 \sqrt{2}}{\sqrt{N_{c}^{2}-4}}
\end{gathered}
$$
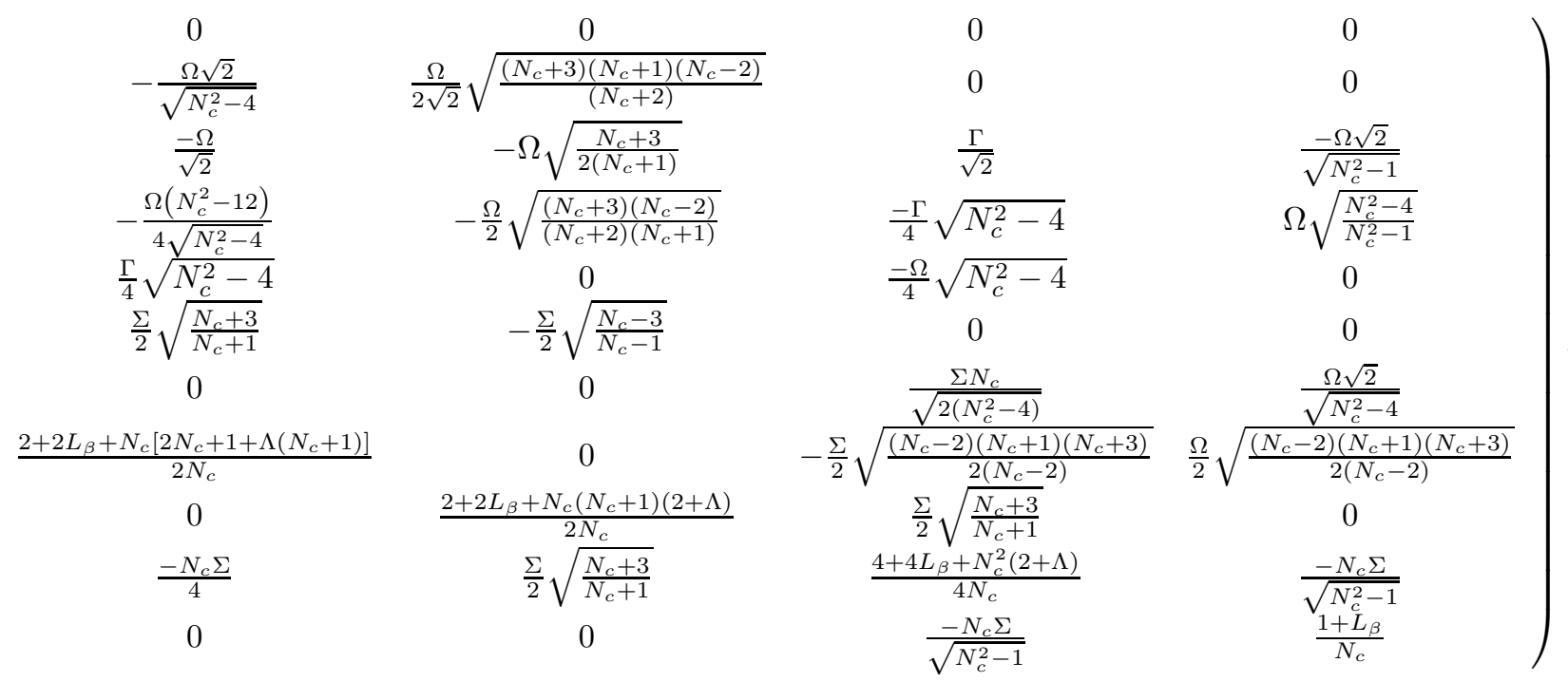


\begin{tabular}{|c|c|c|c|c|c|c|}
\hline$N_{c} \ln \left(v_{15} v_{25}\right)$ & 0 & 0 & 0 & $\frac{1}{\sqrt{2}} \ln \left(\frac{v_{45}}{v_{35}}\right)$ & 0 & 0 \\
\hline 0 & $N_{c} \ln \left(v_{15} v_{25}\right)$ & 0 & 0 & 0 & $\frac{1}{2} \ln \left(\frac{v_{45}}{v_{35}}\right)$ & 0 \\
\hline 0 & 0 & $N_{c} \ln \left(v_{15} v_{25}\right)$ & 0 & 0 & 0 & 0 \\
\hline 0 & 0 & 0 & $\frac{1}{4} N_{c} \ln \left(v_{15}^{2} v_{25}^{2} v_{35} v_{45}\right)$ & $\frac{1}{2} N_{c} \ln \left(\frac{v_{15}}{v_{25}}\right)$ & 0 & 0 \\
\hline$\frac{1}{\sqrt{2}} \ln \left(\frac{v_{45}}{v_{35}}\right)$ & 0 & 0 & $\frac{1}{2} N_{c} \ln \left(\frac{v_{15}}{v_{25}}\right)$ & $\frac{1}{4} N_{c} \ln \left(v_{15}^{2} v_{25}^{2} v_{35} v_{45}\right)$ & 0 & 0 \\
\hline 0 & $\frac{1}{2} \ln \left(\frac{v_{45}}{v_{35}}\right)$ & 0 & 0 & 0 & $N_{c} \ln \left(v_{15} v_{25}\right)$ & \\
\hline 0 & 0 & 0 & 0 & 0 & & $2\left(N_{c}-1\right) \ln \left(v_{15} v_{25}\right)+\ln \left(v_{35} v_{45}\right)$ \\
\hline 0 & 0 & 0 & 0 & $\frac{1}{4} \ln \left(\frac{v_{45}}{v_{35}}\right) \sqrt{N_{c}^{2}-4}$ & $\frac{N_{c} \ln \left(\frac{v_{25}}{v_{15}}\right) \sqrt{2}}{\sqrt{N_{c}^{2}-4}}$ & 0 \\
\hline 0 & 0 & 0 & 0 & 0 & $\ln \left(\frac{v_{15}}{v_{25}}\right) \sqrt{\frac{\left(N_{c}+3\right)\left(N_{c}+1\right)\left(N_{c}-2\right)}{N_{c}+2}}$ & 0 \\
\hline 0 & 0 & $\frac{1}{\sqrt{2}} \ln \left(\frac{v_{45}}{v_{35}}\right)$ & $\frac{\ln \left(\frac{v_{25}}{v_{15}}\right)}{4} \sqrt{N_{c}^{2}-4}$ & 0 & 0 & $\ln \left(\frac{v_{25}}{v_{15}}\right) \sqrt{\frac{N_{c}-3}{N_{c}-1}}$ \\
\hline 0 & 0 & 0 & 0 & 0 & 0 & 0 \\
\hline
\end{tabular}

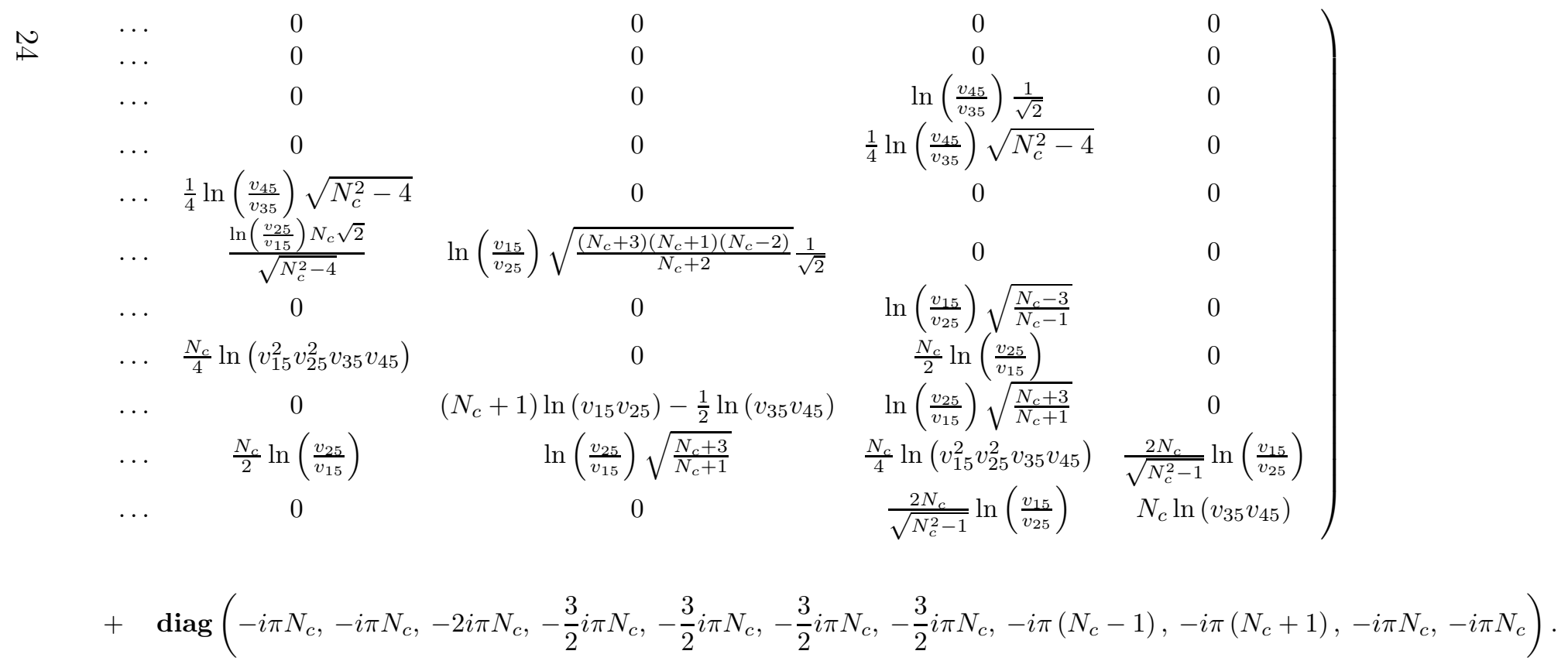


In the next step a special case is considered $p_{3}^{\mu}=p_{4}^{\mu}$. The obtained matrix has a block - diagonal form:

$$
\Gamma_{g g \rightarrow Q \bar{Q} g}\left(p_{3}^{\mu}=p_{4}^{\mu}\right)=\frac{\alpha_{s}}{\pi} T_{R} \times\left(\begin{array}{ccc}
\Gamma_{\mathbf{3} \times \mathbf{3}} & & \\
& \Gamma_{\mathbf{2} \times \mathbf{2}} & \\
& & \Gamma_{\mathbf{6} \times \mathbf{6}}
\end{array}\right)
$$

where

$$
\begin{aligned}
\Gamma_{\mathbf{3} \times \mathbf{3}}= & \frac{1}{N_{c}} \times \operatorname{diag}\left\{1+\left(1-N_{c}^{2}\right) L_{\beta}+N_{c}^{2} \ln \left(v_{15} v_{25}\right)-i \pi N_{c}^{2},\right. \\
& 1+L_{\beta}+N_{c}^{2}\left(1-i \pi+\frac{\Lambda^{\prime}}{2}+\ln \left(v_{15} v_{25}\right)\right), \\
& \left.1+\left(1-N_{c}^{2}\right) L_{\beta}+N_{c}^{2} \ln \left(v_{15} v_{25}\right)-i \pi N_{c}^{2},\right\}, \\
\Gamma_{\mathbf{2} \times \mathbf{2}=} & \left(\begin{array}{cc}
\frac{4+4 L_{\beta}+N_{c}^{2}\left(2-6 i \pi+\Lambda^{\prime}+2 \ln \left(v_{15} v_{25} v_{35}\right)\right)}{4 N_{c}} & -\frac{N_{c}}{4}\left(\Sigma^{\prime}-2 \ln \left(\frac{v_{25}}{v_{15}}\right)\right) \\
-\frac{N_{c}}{4}\left(\Sigma^{\prime}-2 \ln \left(\frac{v_{25}}{v_{15}}\right)\right) & \frac{4+4 L_{\beta}+N_{c}^{2}\left(2-6 i \pi+\Lambda^{\prime}+2 \ln \left(v_{15} v_{25} v_{35}\right)\right)}{4 N_{c}}
\end{array}\right),
\end{aligned}
$$

and 

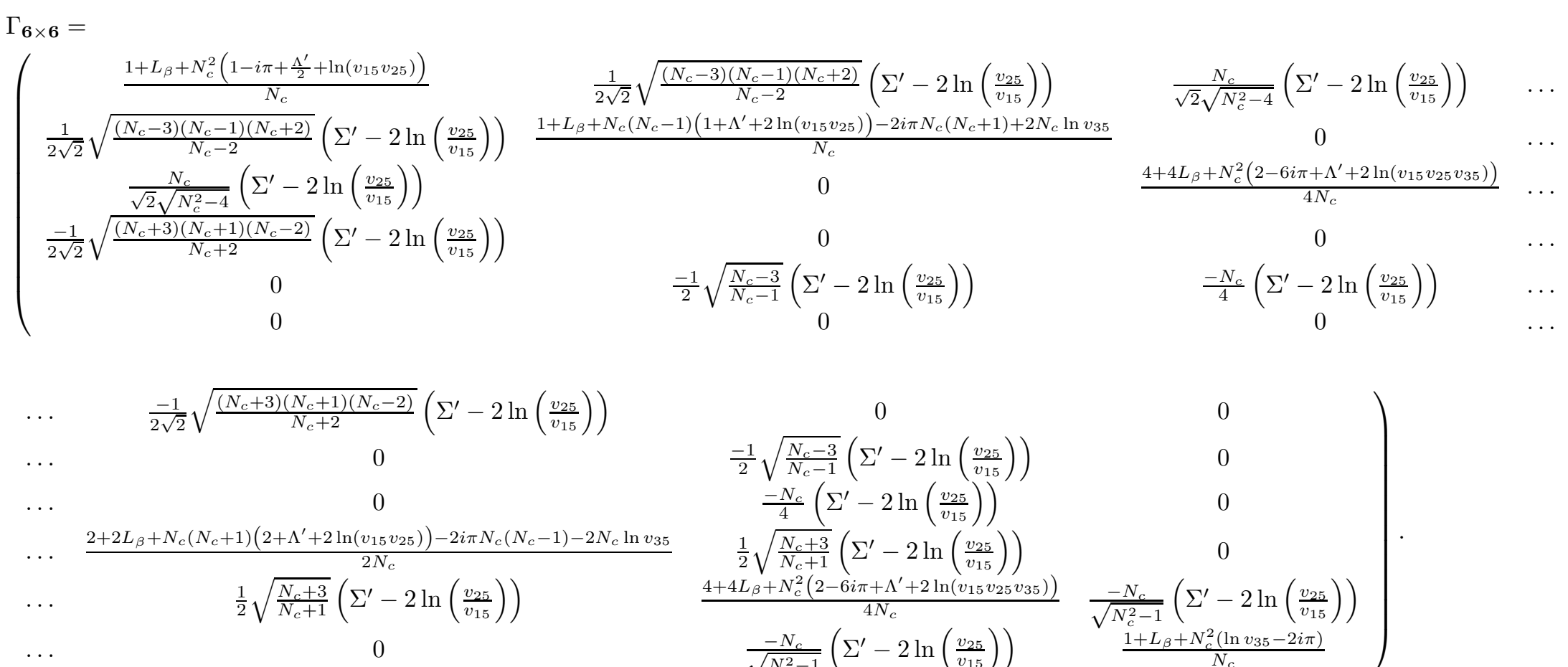

$$
\left.\begin{array}{cc}
0 & 0 \\
\frac{-1}{2} \sqrt{\frac{N_{c}-3}{N_{c}-1}}\left(\Sigma^{\prime}-2 \ln \left(\frac{v_{25}}{v_{15}}\right)\right) & 0 \\
\frac{-N_{c}}{4}\left(\Sigma^{\prime}-2 \ln \left(\frac{v_{25}}{v_{15}}\right)\right) & 0 \\
\frac{1}{2} \sqrt{\frac{N_{c}+3}{N_{c}+1}}\left(\Sigma^{\prime}-2 \ln \left(\frac{v_{25}}{v_{15}}\right)\right) & 0 \\
\frac{4+4 L_{\beta}+N_{c}^{2}\left(2-6 i \pi+\Lambda^{\prime}+2 \ln \left(v_{15} v_{25} v_{35}\right)\right)}{4 N_{c}} & \frac{-N_{c}}{\sqrt{N_{c}^{2}-1}}\left(\Sigma^{\prime}-2 \ln \left(\frac{v_{25}}{v_{15}}\right)\right) \\
\frac{-N_{c}}{\sqrt{N_{c}^{2}-1}}\left(\Sigma^{\prime}-2 \ln \left(\frac{v_{25}}{v_{15}}\right)\right) & \frac{1+L_{\beta}+N_{c}^{2}\left(\ln v_{35}-2 i \pi\right)}{N_{c}}
\end{array}\right) .
$$

For the case $N_{c}=3$, the last block becomes even simpler: $\Gamma_{\mathbf{6} \times \mathbf{6}}=\Gamma_{\mathbf{1} \times \mathbf{1}} \otimes \Gamma_{\mathbf{5} \times \mathbf{5}}$. After performing the limit $\Sigma^{\prime}=0$ matrices take the following form: 


$$
\begin{aligned}
& \Gamma_{g g \rightarrow Q \bar{Q} g}\left(\Sigma^{\prime}=0\right)=\frac{\alpha_{s}}{\pi} T_{R} \times \frac{1}{N_{c}} \times \\
& \operatorname{diag}\left\{1+\left(1-N_{c}^{2}\right) L_{\beta}+N_{c}^{2} \ln \left(v_{15} v_{25}\right)-i \pi N_{c}^{2}, 1+L_{\beta}+N_{c}^{2}\left(1-i \pi+\frac{\Lambda^{\prime}}{2}+\ln \left(v_{15} v_{25}\right)\right),\right. \\
& 1+\left(1-N_{c}^{2}\right) L_{\beta}+N_{c}^{2} \ln \left(v_{15} v_{25}\right)-i \pi N_{c}^{2}, 1+L_{\beta}+\frac{N_{c}^{2}}{4}\left(2-6 i \pi+\Lambda^{\prime}+2 \ln \left(v_{15} v_{25} v_{35}\right)\right), \\
& 1+L_{\beta}+\frac{N_{c}^{2}}{4}\left(2-6 i \pi+\Lambda^{\prime}+2 \ln \left(v_{15} v_{25} v_{35}\right)\right), 1+L_{\beta}+N_{c}^{2}\left(1-i \pi+\frac{\Lambda^{\prime}}{2}+\ln \left(v_{15} v_{25}\right)\right) \\
& 1+L_{\beta}+N_{c}\left(N_{c}-1\right)\left(1+\Lambda^{\prime}+2 \ln \left(v_{15} v_{25}\right)\right)-2 i \pi N_{c}\left(N_{c}+1\right)+2 N_{c} \ln v_{35} \\
& 1+L_{\beta}+\frac{N_{c}^{2}}{4}\left(2-6 i \pi+\Lambda^{\prime}+2 \ln \left(v_{15} v_{25} v_{35}\right)\right), \\
& 1+L_{\beta}+\frac{N_{c}}{2}\left(N_{c}+1\right)\left(2+\Lambda^{\prime}+2 \ln \left(v_{15} v_{25}\right)\right)-i \pi N_{c}\left(N_{c}-1\right)-N_{c} \ln v_{35}, \\
& \left.1+L_{\beta}+\frac{N_{c}^{2}}{4}\left(2-6 i \pi+\Lambda^{\prime}+2 \ln \left(v_{15} v_{25} v_{35}\right)\right), 1+L_{\beta}+N_{c}^{2}\left(\ln v_{35}-2 i \pi\right)\right\}
\end{aligned}
$$

\subsubsection{Analysis of the eigenvalues for $g g \rightarrow Q \bar{Q} g$}

In this subsection, we perform an analogous analysis of the eigensystem for $g g \rightarrow$ $Q \bar{Q} g$ to the case of $q \bar{q} \rightarrow Q \bar{Q} g$. The set of the eigenvalues is richer then in the scattering process of the quark and antiquark due to the larger colour basis. For $\theta=90^{\circ}$ the real parts of the regularized eigenvalues are shown in fig. 5 and the imaginary parts are shown in fig. 6, The singular matrix in $\beta_{34}$ has a form $\frac{i \pi}{\beta_{34}} \times \operatorname{diag}\left(-\frac{2}{3},-\frac{2}{3}, \frac{1}{12}, \frac{1}{12}, \frac{1}{12}, \frac{1}{12}, \frac{1}{12}, \frac{1}{12}, \frac{1}{12}, \frac{1}{12}, \frac{1}{12}\right)$ in this case. One finds also one value of the leading small $\beta$ behaviour of the eigenvalues, which is the same as in the quark channel:

$$
\lambda^{\operatorname{sing}}=6 \log \beta
$$

After the procedure of regularization (analogous to the $q \bar{q}$ scattering case) one can see some similarities for both the scattering angles. In the case $\theta=90^{\circ}$ the eigensystem consists of 6 different eigenvalues. The degenerate eigenvalues are 
$\lambda_{1}=\lambda_{2}, \lambda_{3}=\lambda_{4}=\lambda_{5}=\lambda_{6}$ and $\lambda_{8}=\lambda_{9}$. The real parts of the eigenvalues are nearly constant up to $\beta \approx 0.6$ and all the imaginary parts are constant in whole range of $\beta$. The results for $\theta=30^{\circ}$ are shown in fig. 7 (the real parts of eigenvalues) and in fig. 8 (the imaginary parts of eigenvalues). The singular part of the eigenvalues at $\theta=30^{\circ}$ is the same as for $\theta=90^{\circ}$. The degeneracy of the eigensystem is lower (the degeneracy between eigenvalues 4,5,6,7 is reduced to the separate degeneracy $\lambda_{3}=\lambda_{4}$ and $\lambda_{5}=\lambda_{6}$ ). The real parts of eigenvalues are nearly flat for $\beta<0.5$ then they grow rapidly. $\operatorname{Im}\left(\lambda_{10}^{30^{\circ}}\right)\left(\operatorname{Im}\left(\lambda_{11}^{30^{\circ}}\right)\right)$ is a growing (decreasing) function of $\beta$. The imaginary parts of the remaining eigenvalues are constant.

Moreover, comparing the behaviour of the eigensystem of SAD matrices for processes $q \bar{q} \rightarrow Q \bar{Q} g$ and $g g \rightarrow Q \bar{Q} g$ one finds some similarities. For example, at $\theta=90^{\circ}$ there is a constant behaviour in $\beta$ for the imaginary part of eigenvalues in both reactions. When the kinematic configuration becomes less symmetrical (the $\theta=30^{\circ}$ case) the set of eigenvalues with a flat $\beta$-dependence is reduced.

\section{Discussion and summary}

In this paragraph we compare the calculated regularized eigenvalues $\lambda_{i}^{\text {reg }}$ of the SAD matrices to the SAD eigenvalues for processes $q \bar{q} \rightarrow Q \bar{Q}$ and $g g \rightarrow Q \bar{Q}$ in the small $\beta$ region. Note that the full eigenvalues for $2 \rightarrow 3$ processes contain in addition to the regular parts a negative singular term $6 \log \beta$ for the $q \bar{q}$ and gg channel. The logarithmic terms combine with the dominant regular terms into 

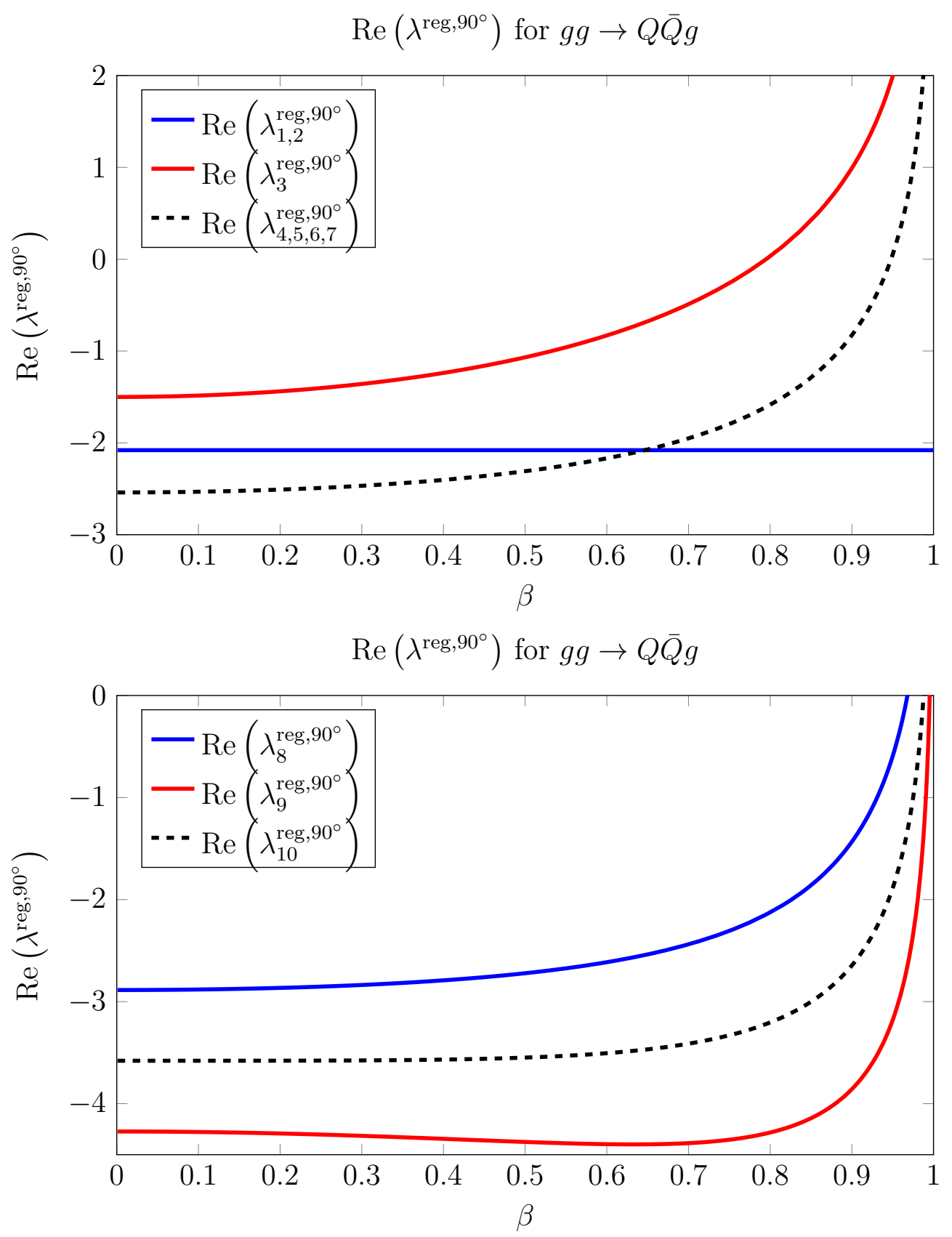

Figure 5. The real parts of the regularized eigenvalues of $\tilde{\Gamma}_{S}$ for $g g \rightarrow Q \bar{Q} g$ at $\theta=90^{\circ}$. 


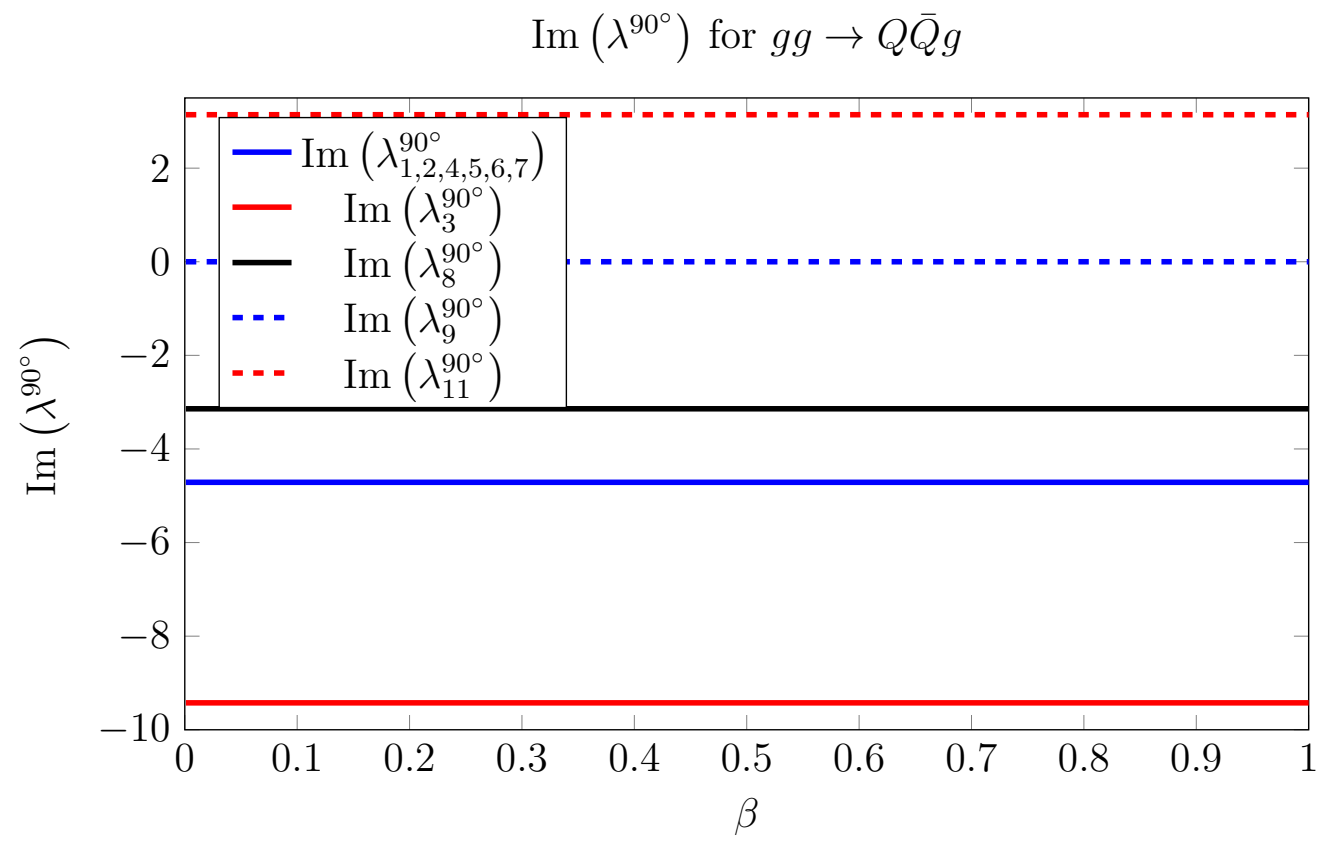

Figure 6. The imaginary parts of the eigenvalues of $\tilde{\Gamma}_{S}$ for $g g \rightarrow Q \bar{Q} g$ at $\theta=90^{\circ}$.

even larger negative terms of the eigenvalues, that is they lead to stronger effects of gluon radiation. For the process $q \bar{q} \rightarrow Q \bar{Q}$ the real parts of the two at $\beta \rightarrow 0$ tend to $-1.5 \frac{\alpha_{s}}{\pi}$ and 0 . Recall that for the case of $q \bar{q} \rightarrow Q \bar{Q} g$ the largest (negative) SAD eigenvalue reads $\operatorname{Re}\left(\lambda^{\mathrm{reg}, 90^{\circ}}\right)=-2.5 \frac{\alpha_{s}}{\pi}\left(\right.$ for $\left.\theta=90^{\circ}\right)$ and $\operatorname{Re}\left(\lambda^{\mathrm{reg}, 30^{\circ}}\right)=-5 \frac{\alpha_{s}}{\pi}$ (for $\theta=30^{\circ}$ ). It means that the effect of soft gluon radiation for $q \bar{q} \rightarrow Q \bar{Q} g$ is almost two times stronger (the $90^{\circ}$ case) or three times larger (the $30^{\circ}$ case). In the gluonic case the radiation effects are even stronger. For $g g \rightarrow Q \bar{Q} g$ we obtained $\operatorname{Re}\left(\lambda^{\mathrm{reg}, 90^{\circ}}\right)=-4 \frac{\alpha_{s}}{\pi}\left(\right.$ for $\left.\theta=90^{\circ}\right)$ and $\operatorname{Re}\left(\lambda^{\text {reg, } 30^{\circ}}\right)=-8 \frac{\alpha_{s}}{\pi}\left(\right.$ for $\left.\theta=30^{\circ}\right)$, so the radiation is enhanced by factors three and five correspondingly with respect to the process $g g \rightarrow Q \bar{Q}$. The imaginary parts of eigenvalues cancel out in the regime $\beta \rightarrow 0$, so we will not discuss them. These results imply that the soft gluon radiation is a source of enhanced corrections for the heavy quark pair production in association with a gluon jet. 

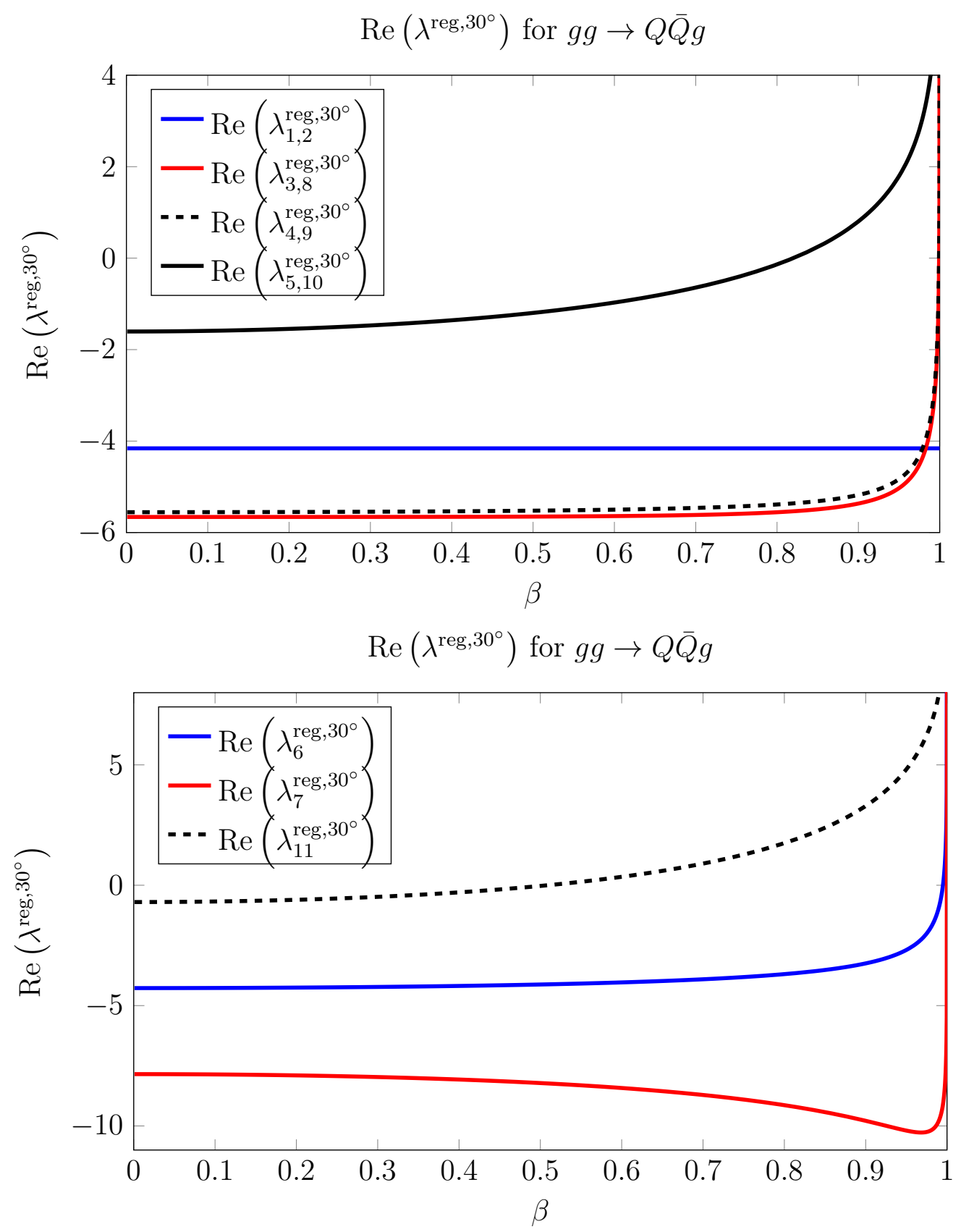

Figure 7. The real parts of the regularized eigenvalues of $\tilde{\Gamma}_{S}$ for $g g \rightarrow Q \bar{Q} g$ at $\theta=30^{\circ}$. 


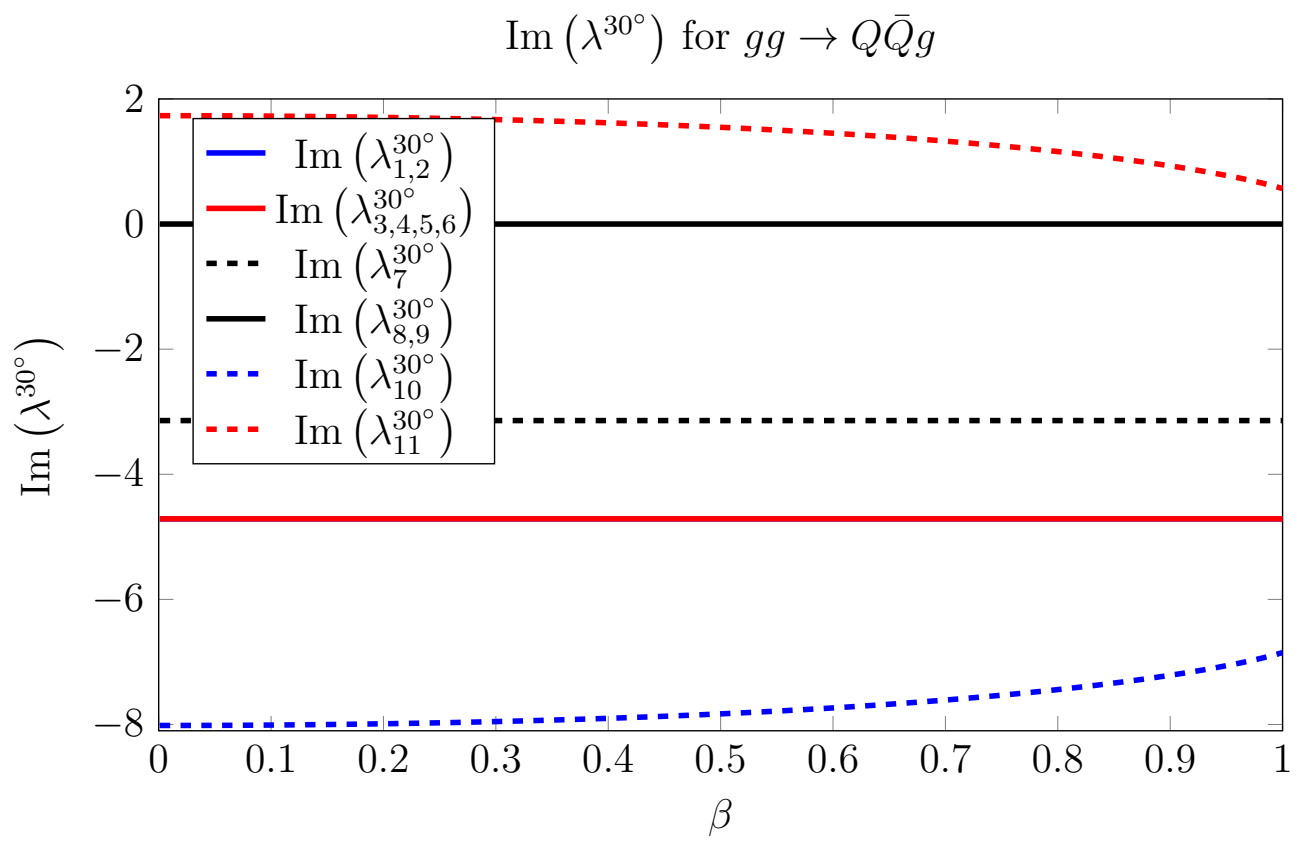

Figure 8. The imaginary parts of the eigenvalues of $\tilde{\Gamma}_{S}$ for $g g \rightarrow Q \bar{Q} g$ at $\theta=30^{\circ}$.

In this paper we have derived the one-loop soft anomalous dimension matrices for $q \bar{q} \rightarrow Q \bar{Q} g$ and $g g \rightarrow Q \bar{Q} g$. We presented the SAD matrices for an arbitrary scattering angle $\theta$ of a clustered pair of heavy quark and antiquark with respect to the incoming parton axis in the CMS frame. We also analyzed the spectrum of the eigenvalues of the SAD matrices in details for two kinematic configurations $\theta=90^{\circ}$ and $30^{\circ}$, performing explicit numerical calculations of the SAD eigenvalues. The obtained results are a step towards implementing the soft resummation procedure for $Q \bar{Q}$ - jet production in hadron colliders, and improving accuracy of theoretical predictions.

\section{Acknowledgments}

The author would like to thank Prof. L. Motyka for the help of preparing this paper, Prof. M. Praszałowicz for valuable comments on the manuscript, Prof. A. 
Kulesza for the discussion, Prof. M. Sjödahl and R. Schäfer for the correspondence.

This work was supported by the Polish NCN grant DEC-2014/13/B/ST2/02486.

\section{References}

[1] J. C. Collins, D. E. Soper and G. F. Sterman, "Factorization for Short Distance Hadron - Hadron Scattering," Nucl. Phys. B 261 (1985) 104. doi:10.1016/0550-3213(85)90565-6

[2] N. Kidonakis and G. F. Sterman, "Resummation for QCD hard scattering," Nucl. Phys. B 505 (1997) 321 doi:10.1016/S0550-3213(97)00506-3 hep-ph/9705234.

[3] G. F. Sterman, "Summation of Large Corrections to Short Distance Hadronic Cross-Sections," Nucl. Phys. B 281, 310 (1987). doi:10.1016/05503213(87)90258-6

[4] S. Catani and L. Trentadue, "Resummation of the QCD Perturbative Series for Hard Processes," Nucl. Phys. B 327 (1989) 323. doi:10.1016/05503213(89)90273-3

[5] N. Kidonakis, G. Oderda and G. F. Sterman, "Evolution of color exchange in QCD hard scattering," Nucl. Phys. B 531 (1998) 365 doi:10.1016/S05503213(98)00441-6 [hep-ph/9803241].

[6] R. Bonciani, S. Catani, M. L. Mangano and P. Nason, "NLL resummation of the heavy quark hadroproduction cross-section," Nucl. Phys. B 529 (1998) 424 Erratum: [Nucl. Phys. B 803 (2008) 234] doi:10.1016/j.nuclphysb.2008.06.006, 10.1016/S0550-3213(98)00335-6 hep-ph/9801375|. 10.1016/S0550-3213(98)00335-6;

[7] N. Kidonakis and R. Vogt, "Next-to-next-to-leading order soft gluon corrections in top quark hadroproduction," Phys. Rev. D 68 (2003) 114014 doi:10.1103/PhysRevD.68.114014 |hep-ph/0308222].

[8] M. Czakon, A. Mitov and G. F. Sterman, "Threshold Resummation for TopPair Hadroproduction to Next-to-Next-to-Leading Log," Phys. Rev. D 80 (2009) 074017 doi:10.1103/PhysRevD.80.074017 [arXiv:0907.1790 [hep-ph]].

[9] V. Ahrens, A. Ferroglia, M. Neubert, B. D. Pecjak and L. L. Yang, "Renormalization-Group Improved Predictions for Top-Quark Pair Production at Hadron Colliders," JHEP 1009 (2010) 097 doi:10.1007/JHEP09(2010)097 [arXiv:1003.5827 [hep-ph]]. 
[10] M. Cacciari, M. Czakon, M. Mangano, A. Mitov and P. Nason, next-to-nextto-leading logarithmic soft-gluon resummation," Phys. Lett. B 710 (2012) 612 doi:10.1016/j.physletb.2012.03.013 [arXiv:1111.5869 [hep-ph]].

[11] N. Kidonakis, "Next-to-next-to-leading-order collinear and soft gluon corrections for t-channel single top quark production," Phys. Rev. D 83 (2011) 091503 doi:10.1103/PhysRevD.83.091503 [arXiv:1103.2792 [hep-ph]].

[12] M. Czakon, P. Fiedler and A. Mitov, "Total Top-Quark Pair-Production Cross Section at Hadron Colliders Through $O\left(\alpha \frac{4}{S}\right)$," Phys. Rev. Lett. 110 (2013) 252004 doi:10.1103/PhysRevLett.110.252004 [arXiv:1303.6254 [hep-ph]].

[13] M. Beneke, P. Falgari, S. Klein and C. Schwinn, "Hadronic top-quark pair production with NNLL threshold resummation," Nucl. Phys. B 855 (2012) 695 doi:10.1016/j.nuclphysb.2011.10.021 [arXiv:1109.1536 [hep-ph]].

[14] A. Kulesza and L. Motyka, "Threshold resummation for squark-antisquark and gluino-pair production at the LHC," Phys. Rev. Lett. 102, 111802 (2009) doi:10.1103/PhysRevLett.102.111802 [arXiv:0807.2405 [hep-ph]].

[15] A. Kulesza and L. Motyka, "Soft gluon resummation for the production of gluino-gluino and squark-antisquark pairs at the LHC," Phys. Rev. D 80 (2009) 095004 doi:10.1103/PhysRevD.80.095004 [arXiv:0905.4749 [hep-ph]].

[16] W. Beenakker, S. Brensing, M. n. Kramer, A. Kulesza, E. Laenen, L. Motyka and I. Niessen, "Squark and Gluino Hadroproduction," Int. J. Mod. Phys. A 26 (2011) 2637 doi:10.1142/S0217751X11053560 [arXiv:1105.1110 [hep-ph]].

[17] W. Beenakker, S. Brensing, M. Kramer, A. Kulesza, E. Laenen and I. Niessen, "NNLL resummation for squark-antisquark pair production at the LHC," JHEP 1201 (2012) 076 doi:10.1007/JHEP01(2012)076 arXiv:1110.2446 [hep$\mathrm{ph}]$.

[18] W. Beenakker, C. Borschensky, M. Kramer, A. Kulesza, E. Laenen, $\mathrm{V}$. Theeuwes and S. Thewes, "NNLL resummation for squark and gluino production at the LHC," JHEP 1412 (2014) 023 doi:10.1007/JHEP12(2014)023 arXiv:1404.3134 [hep-ph]].

[19] W. Beenakker, C. Borschensky, R. Heger, M. Kramer, A. Kulesza and E. Laenen, "NNLL resummation for stop pair-production at the LHC," JHEP 1605 (2016) 153 doi:10.1007/JHEP05(2016)153 [arXiv:1601.02954 [hep-ph]].

[20] S. Catani, D. de Florian, M. Grazzini and P. Nason, "Soft gluon resummation for Higgs boson production at hadron colliders," JHEP 0307 (2003) 028 doi:10.1088/1126-6708/2003/07/028 hep-ph/0306211.

[21] M. Bonvini, S. Marzani, C. Muselli and L. Rottoli, "On the Higgs cross section at $\mathrm{N}^{3} \mathrm{LO}+\mathrm{N}^{3} \mathrm{LL}$ and its uncertainty," JHEP 1608 (2016) 105 doi:10.1007/JHEP08(2016)105 [arXiv:1603.08000 [hep-ph]]. 
[22] N. Kidonakis, "Two-loop soft anomalous dimensions for single top quark associated production with a W- or H-," Phys. Rev. D 82 (2010) 054018 doi:10.1103/PhysRevD.82.054018 [arXiv:1005.4451 [hep-ph]].

[23] A. Kulesza, L. Motyka, T. Stebel and V. Theeuwes, "Soft gluon resummation for associated $t \bar{t} H$ production at the LHC," JHEP 1603 (2016) 065 doi:10.1007/JHEP03(2016)065 [arXiv:1509.02780 [hep-ph]].

[24] A. Broggio, A. Ferroglia, B. D. Pecjak, A. Signer and L. L. Yang, "Associated production of a top pair and a Higgs boson beyond NLO," JHEP 1603 (2016) 124 doi:10.1007/JHEP03(2016)124 [arXiv:1510.01914 [hep-ph]].

[25] A. Broggio, A. Ferroglia, B. D. Pecjak and L. L. Yang, "NNLL resummation for the associated production of a top pair and a Higgs boson at the LHC," JHEP 1702 (2017) 126 doi:10.1007/JHEP02(2017)126 arXiv:1611.00049 [hep-ph]].

[26] A. Kulesza, L. Motyka, T. Stebel and V. Theeuwes, "Associated $t \bar{t} H$ production at the LHC: theoretical predictions at NLO+NNLL accuracy," arXiv:1704.03363 [hep-ph].

[27] M. Sjödahl, "Color evolution of $2 \rightarrow 3$ processes," JHEP 0812, 083 (2008) doi:10.1088/1126-6708/2008/12/083 [arXiv:0807.0555 [hep-ph]].

[28] M. Sjödahl, "ColorMath - A package for color summed calculations in SU(Nc)," Eur. Phys. J. C 73, no. 2, 2310 (2013) doi:10.1140/epjc/s10052013-2310-4 [arXiv:1211.2099 [hep-ph]].

[29] T. Becher and M. Neubert, "Infrared singularities of scattering amplitudes in perturbative QCD," Phys. Rev. Lett. 102 (2009) 162001 Erratum: [Phys. Rev. Lett. 111 (2013) no.19, 199905] doi:10.1103/PhysRevLett.102.162001, 10.1103/PhysRevLett.111.199905 arXiv:0901.0722 [hep-ph]]. 10.1103/PhysRevLett.111.199905;

[30] T. Becher and M. Neubert, "On the Structure of Infrared Singularities of Gauge-Theory Amplitudes," JHEP 0906 (2009) 081 Erratum: [JHEP 1311 (2013) 024] doi:10.1088/1126-6708/2009/06/081, 10.1007/JHEP11(2013)024 arXiv:0903.1126 [hep-ph]]. 10.1007/JHEP11(2013)024;

[31] T. Becher and M. Neubert, "Infrared singularities of QCD amplitudes with massive partons," Phys. Rev. D 79 (2009) 125004 Erratum: [Phys. Rev. D 80 (2009) 109901] doi:10.1103/PhysRevD.79.125004, 10.1103/PhysRevD.80.109901 [arXiv:0904.1021 [hep-ph]]. 10.1103/PhysRevD.80.109901;

[32] M. H. Seymour and M. Sjödahl, "Symmetry of anomalous dimension matrices explained," JHEP 0812 (2008) 066 doi:10.1088/1126-6708/2008/12/066 arXiv:0810.5756 [hep-ph]].

[33] R. Schäfer, Bachelor Thesis, "The colour evolution of the $q \bar{q} g$ and $Q \bar{Q} g$ production in the quark channel," 2017 